Barker, P.F., Camerlenghi, A., Acton, G.D., and Ramsay, A.T.S. (Eds.)

Proceedings of the Ocean Drilling Program, Scientific Results Volume 178

\section{DATA Report: Diffuse Spectral Reflectance Data from Rise Sites 1095, 1096, AND 1101 AND PALMER DeEP Sites 1098 AND 1099 (LEG 178, Western Antarctic Peninsula)'}

T.C.W. Wolf-Welling, ${ }^{2}$ Ellen A. Cowan, ${ }^{3}$ J. Daniels, ${ }^{4}$ N. Eyles, ${ }^{5}$ A. Maldonado, ${ }^{6}$ and Carol J. Pudsey ${ }^{7}$

\section{INTRODUCTION}

The routine use of spectrophotometry on the sediment surfaces of archive halves of each section during the onboard sedimentological core description process is a great stride toward development of real-time noninvasive characterization of deep-sea sediments. Spectral reflectance data have been used so far for mineral composition studies as well as for lithostratigraphic correlation between sites (Balsam and Deaton, 1991; Balsam et al., 1997; Mix et al., 1995; Ortiz et al., 1999). Their results demonstrate that spectrophotometry can estimate $\mathrm{CaCO}_{3}$ content by using the 4.65-, 5.25-, and 5.55- $\mu \mathrm{m}$ wavelength spectrums. A detailed overview of various other noninvasive methods is given in Ortiz and Rack (1999).

The purpose of this study is to test whether spectrophotometry in the visible band can be used as a tool to gather further information about grain-size variation, sorting, compaction, and porosity, which are directly linked to the sedimentation process. From remote sensing data analyses, it is known that diffuse spectral reflectance data in the visible band in the wavelength window of 7.0-6.5 $\mu \mathrm{m}$ are sensitive to grainsize variations. It appears that a relationship between grain size and signal absorption exists only in this wavelength window. (e.g., Clark, 1999; Gaffey, 1986; Gaffey et al., 1993). Variations in grain size during a
${ }^{1}$ Wolf-Welling, T.C.W., Cowan, E.A., Daniels, J., Eyles, N., Maldonado, A., and Pudsey, C.J., 2001. Data report: Diffuse spectral reflectance data from rise Sites 1095, 1096, and 1101 and Palmer Deep Sites 1098 and 1099 (Leg 178, western Antarctic Peninsula.) In Barker, P.F., Camerlenghi, A., Acton, G.D., and Ramsay, A.T.S. (Eds.), Proc. ODP, Sci. Results, 178, 1-22 [Online]. Available from World Wide Web: $<$ http://www-odp.tamu.edu/ publications/178_SR/VOLUME/ CHAPTERS/SR178_21.PDF $>$. [Cited YYYY-MM-DD]

${ }^{2}$ Institute for Geosciences at Christian Albrechts University, Kiel, Olshausenstrasse 40, 24118 Kiel, Germany.twolf@geomar.de

${ }^{3}$ Department of Geology, Appalachian State University, Boone NC 28608, USA.

${ }^{4}$ School of Earth Sciences, University of Melbourne, Parkville VIC 3052, Australia.

${ }^{5}$ Environmental Earth Sciences, University of Toronto at Scarborough, 1265 Military Trail, Scarborough ON M1C 1A4, Canada.

${ }^{6}$ Instituto Andaluz de Ciencias de la Tierra, CSIC, Universidad de Granada, Facultad de Ciencias, Fuentenueve s/n, 18002 Granada, Spain.

${ }^{7}$ British Antarctic Survey, High Cross, Madingley Road, Cambridge CB3 OET, United Kingdom.

Initial receipt: 17 October 2000 Acceptance: 2 July 2001

Web publication: 16 November 2001 Ms 178SR-225 
sedimentation process are linked to depositional energy, which affects sorting, compaction, and porosity of sediment deposits. As an example, we study here the spectrophotometric data of the sedimentary sequence of Hole 1098C, which was deposited under widely varying environmental conditions. Alternating turbidite and finely laminated sediments were recovered from Hole 1098C. The turbidites are related to a high depositional energy environment; the finely laminated sediments are related to a low depositional energy environment. Data from Hole 1098C were therefore used to test whether the spectral reflectance data can provide a proxy for these different depositional environments.

\section{METHODS}

Spectral reflectance was routinely measured for all Leg 178 sites at evenly spaced intervals of $5 \mathrm{~cm}$ (2-cm intervals were used for Sites 1098 and 1099) downhole using a Minolta spectrophotometer CM 2002. The basic flow operation in the illuminating/viewing system of the CM 2002 during a measurement is as follows: photon energy from a pulsed xenon arc lamp is thoroughly diffused inside an integrated sphere and provides even illumination over the area of the specimen surface to be measured. The energy reflected from the specimen surface is transmitted by optical fiber cable to spectral sensor 1 . At the same time, the energy inside the integrated sphere is transmitted to spectral sensor 2. By using two spectral sensors and measuring both the energy that illuminates the specimen surface and the energy reflected by the specimen surface, the effects of variations in spectral characteristics or intensity of illumination is eliminated. The reflected energy is divided by the wavelength from 4 to $7 \mu \mathrm{m}$ at a $0.1-\mu \mathrm{m}$ pitch before striking spectral sensor 1. The sensor converts the received energy distribution pattern into electrical current proportional to the intensity of the energy, then passes the signal to the analog control circuit, where it is subsequently processed in an integrated microcomputer and sent to a storage system via an RS-232C interface. The CM 2002 has an internal software calibration routine and was regularly calibrated against an external light trap and a white $\left(\mathrm{BaSO}_{4}\right.$ standard) ceramic tile cap.

Measurements were taken as soon as possible after the cores were split to minimize redox-associated color changes that occur when deepsea sediments are exposed to the atmosphere. The measured diffuse spectral reflectance values (4-7 $\mu \mathrm{m} ; 0.1-\mu \mathrm{m}$ pitch) are also used to collect information on sediment color. Therefore, the measured reflectance of a specific spectral energy distribution under standardized conditions is compared with the three basic colors, red, green, and blue. The result of the comparison is expressed as X, Y, and Z, respectively, and called the tristimulus values. The tristimulus values $\mathrm{X}, \mathrm{Y}$, and $\mathrm{Z}$ are converted to the CIELAB system, which provides values called $L^{*}, a^{*}$, and $b^{*}$, where $L^{*}$ is the lightness parameter and $a^{*}$ and $b^{*}$ represent the chromaticity parameters. Additionally, the CIELAB parameters were converted to the Munsell color code. As an aid to defining lithostratigraphic changes during the cruise, the CIELAB system parameters were used to describe changes in the color of cored sediments during Leg 178. Further details are given in the "Explanatory Notes" chapter of the Leg 178 Initial Reports volume (Barker, Camerlenghi, Acton, et al., 1999).

Data sets for each hole consist of sample codes indicating the level at which the measurement was taken, curator depth in meters below seafloor (mbsf), compressed curator depth (mbsf), CIELAB parameter $\left(\mathrm{L}^{*}\right.$, 
$a^{*}$, and $\left.b^{*}\right)$, Munsell color code, and the values for the spectral distribution (4-7 $\mu \mathrm{m} ; 0.1-\mu \mathrm{m}$ pitch).

\section{RESULTS}

Data used for the study presented here are given in Table T1. Spectral reflectance data measured on board for each hole are given in Tables T2, T3, T4, T5, T6, T7, T8, T9, T10, T11, T12, T13, T14, and T15.

For the investigations performed, the data quality was controlled by the ability of the CM 2002 to convert the spectral signal into color data (CIELAB parameters and/or Munsell color). Spectral distribution measurements that were not convertible to color values by the CM 2002, due to cracks or voids in the measured section, were removed from the data set. Also, all core catcher measurements were removed from the data set to gain a data set comparable to other physical properties data. Typically, $<5 \%$ of the data was removed.

Results for Hole 1098C demonstrate that the spectrum of reflectance data (wavelength window $=7.0-6.5 \mu \mathrm{m}$ ) of samples documents systematic differences in signal absorption (Fig. F1); signal absorption is higher in the finely laminated intervals and lower in intervals reported to be turbidites.

As a second step, the variances $\left(\mathrm{s}^{2}\right)$ of the reflectance data at wavelengths between 7.0 and $6.5 \mu \mathrm{m}$ were calculated to get a proxy for the degree of signal absorption by using Equation 1, as follows:

$$
s^{2}=1(n-1)^{-1}\left\{\sum_{i=1}^{n} x_{i}^{2}-\left[\left(1(n)^{-1}\right)\left(\sum_{i=1}^{n} x_{i}\right)^{2}\right]\right\}
$$

Results show that high variance values reflect sediment intervals that are finely laminated and related to a low depositional energy level, whereas low variance values reflect turbiditic sediment intervals related to a high depositional energy level (Fig. F2). Differences in variance values are small, but they are consistent and correlate with the two endmembers of the depositional environments. They are therefore able to characterize sediments deposited at different deposition energy levels vs. depth, as shown for Hole 1098C in Figure F2.

The reasons for these differences are not fully understood at the moment. Its known that finer grain sizes have a lower absorption than coarse grain sizes at wavelengths between 7.0 and $6.5 \mu \mathrm{m}$ (e.g. Clark, 1999). It is also obvious that even small changes in the direction of lower porosity values will lead to a lower absorption, and vice versa.

In this case, two factors must be taken into account during intervals of high deposition energy levels (turbidite facies): (1) the sorting of turbidites is generally low, and (2) because of the higher deposition energy intervals, turbidite sequences consist of a broader grain-size spectrum and are likely to be more compacted during sedimentation, resulting in a slight reduction in porosity. Both factors will result in low variance values of the measured reflectance in the wavelength window (7.0-6.5 $\mu \mathrm{m})$. On the other hand, for intervals of lower deposition energy levels (finely laminated facies), one would expect good sorting into certain grain-size fractions and a lower degree of compaction during the sedimentation process. As a result, variance values will be high.

In summary, diffuse spectral reflectance data provide valuable information about sediment deposition. This data analysis shows that on a
T1. Spectral reflectance data, Hole 1098C, p. 8.

T2. Spectral reflectance data, Hole 1095A, p. 9.

T3. Spectral reflectance data, Hole 1095B, p. 10.

T4. Spectral reflectance data, Hole 1095C, p. 11.

T5. Spectral reflectance data, Hole 1095D, p. 12.

T6. Spectral reflectance data, Hole 1096A, p. 13.

T7. Spectral reflectance data, Hole 1096B, p. 14.

T8. Spectral reflectance data, Hole 1096C, p. 15.

T9. Spectral reflectance data, Hole 1098A, p. 16.

T10. Spectral reflectance data, Hole 1098B, p. 17.

T11. Spectral reflectance data, Hole 1098C, p. 18.

T12. Spectral reflectance data, Hole 1099A, p. 19.

T13. Spectral reflectance data, Hole 1099B, p. 20.

T14. Spectral reflectance data, Hole 1101A, p. 21. 
T.C.W. WOLF-WELLING ET AL.

high-resolution scale, it documents changes in the sedimentation environment as recorded in the two different facies types in Hole 1098C.

\section{ACKNOWLEDGMENTS}

This research used samples and/or data provided by the Ocean Drilling Program (ODP). ODP is sponsored by the U.S. National Science Foundation (NSF) and participating countries under management of Joint Oceanographic Institutions (JOI), Inc. Funding for this research was provided by SPP DSDP/ODP grant Sta 401/6-1/2 of Deutsche Forschungsgemeinschaft.

We gratefully acknowledge the work of the captain, crew members, technical staff, and the co-chiefs and staff scientist of Leg 178, who not only brought the JOIDES Resolution to Antarctica, but also kept us in good spirits during this time. We thank our families and friends for patiently enduring our absence during the cruise and for assistance in preparing this manuscript. We thank A.J. Nederbragt for comments during the review process.
T15. Spectral reflectance data, Hole 1103A, p. 22.

F1. Hole 1098C spectral reflectance data, p. 6.

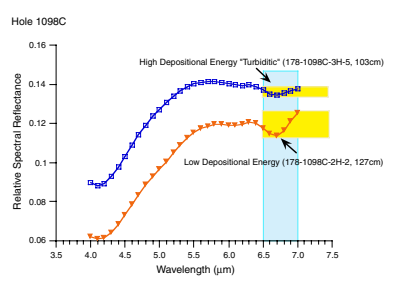

F2. Hole 1098C spectral reflectance variance, p. 7 .

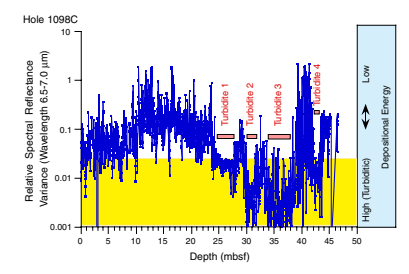




\section{REFERENCES}

Balsam, W.L., Damuth, J.E., and Schneider, R.R., 1997. Comparison of shipboard vs. shore-based spectral data from Amazon-Fan Cores: implications for interpreting sediment composition. In Flood, R.D., Piper, D.J.W., Klaus, A., and Peterson, L.C. (Eds.), Proc. ODP, Sci. Results, 155, 193-215 [CD-ROM]. Available from: Ocean Drilling Program, Texas A\&M University, College Station, TX 77845-9547, U.S.A.

Balsam, W.L., and Deaton, B.C., 1991. Sediment dispersal in the Atlantic Ocean: evaluation by visible light spectra. Rev. Aquat. Sci., 4:411-447.

Barker, P.F., Camerlenghi, A., Acton, G.D., et al., 1999. Proc. ODP, Init. Repts., 178: College Station, TX (Ocean Drilling Program).

Clark, R.N., 1999. Spectroscopy of rocks and minerals and principles of spectroscopy. In Rencz, A.N., and Ryerson, R.A. (Eds.), Manual of Remote Sensing (Vol. 3): Remote Sensing for the Earth Sciences (3rd Edition): New York (Wiley \& Sons), 728.

Gaffey, S.J., 1986. Spectral reflectance of carbonate minerals in the visible and near infrared (0.35 to 2.55 microns): calcite, aragonite, and dolomite. Am. Mineral., 71:151-162.

Gaffey, S.J., McFadden, L.A., Nash, D., and Pieters, C.M., 1993. Ultraviolet, visible, and near infrared reflectance spectroscopy: laboratory spectra of geologic materials. In Pieters, C.M., and Englert, P.A.J. (Eds.), Remote Geochemical Analysis: Elemental and Mineralogical Composition: Cambridge (Cambridge Univ. Press), 43-78.

Mix, A.C., Harris, S.E., and Janecek, T.R., 1995. Estimating lithology from nonintrusive reflectance spectra: Leg 138. In Pisias, N.G., Mayer, L.A., Janecek, T.R., PalmerJulson, A., and van Andel, T.H. (Eds.), Proc. ODP, Sci. Results, 138: College Station, TX (Ocean Drilling Program), 413-427.

Ortiz, J., Mix, A.C., Harris, S., and O'Connell, S., 1999. Diffuse spectral reflectance as a proxy for percent carbonate content in North Atlantic sediments. Paleoceanography, 14:171-186.

Ortiz, J.D., and Rack, F.R., 1999. Non-invasive sediment monitoring methods: current and future tools for high-resolution climate studies. In Abrantes, F., and Mix, A. (Eds.), Reconstructing Ocean History: A Window to the Future: New York (Kluwer Academic/Plenum Publishers), 343-380. 
T.C.W. WOLF-WELLING ET AL.

Data Report: Diffuse Spectral Reflectance Data

Figure F1. Two examples of the spectrum of reflectance data in Hole 1098C. In the wavelength window of 7.0-6.5 $\mu \mathrm{m}$, the absorption of the signal is different in intervals of low and high energy depositional environment, respectively.

Hole 1098C

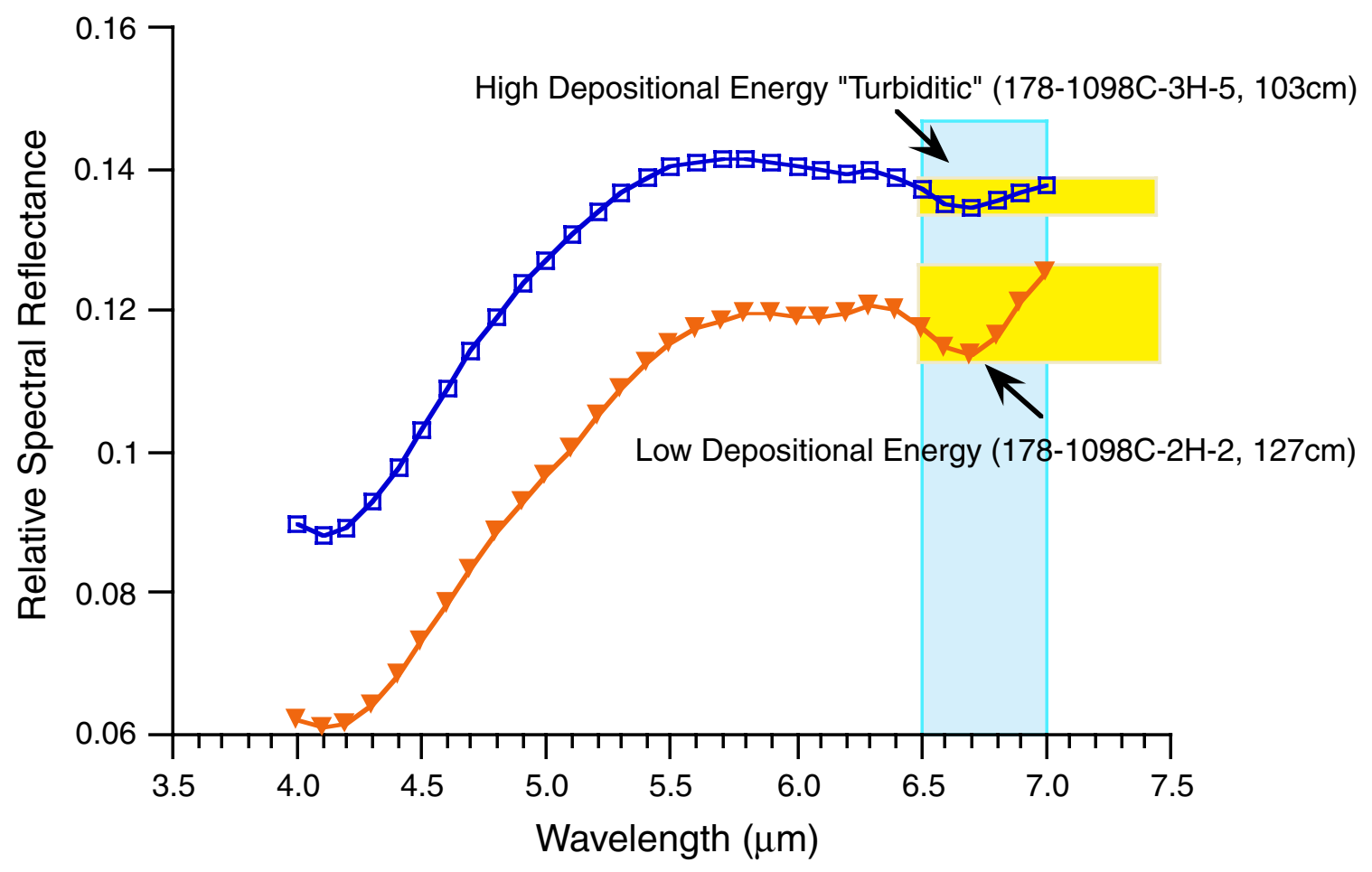


T.C.W. Wolf-Welling ET AL.

DAta Report: Diffuse Spectral Reflectance Data

Figure F2. The variance of the measurements taken in the wavelength window $(7.0-6.5 \mu \mathrm{m})$ as an indicator for the character of signal absorption is shown vs. depth for Hole 1098C. Low variance values document intervals of a high depositional energy facies (turbidites 1 to 4 ), whereas high variance values document intervals low depositional energy facies.

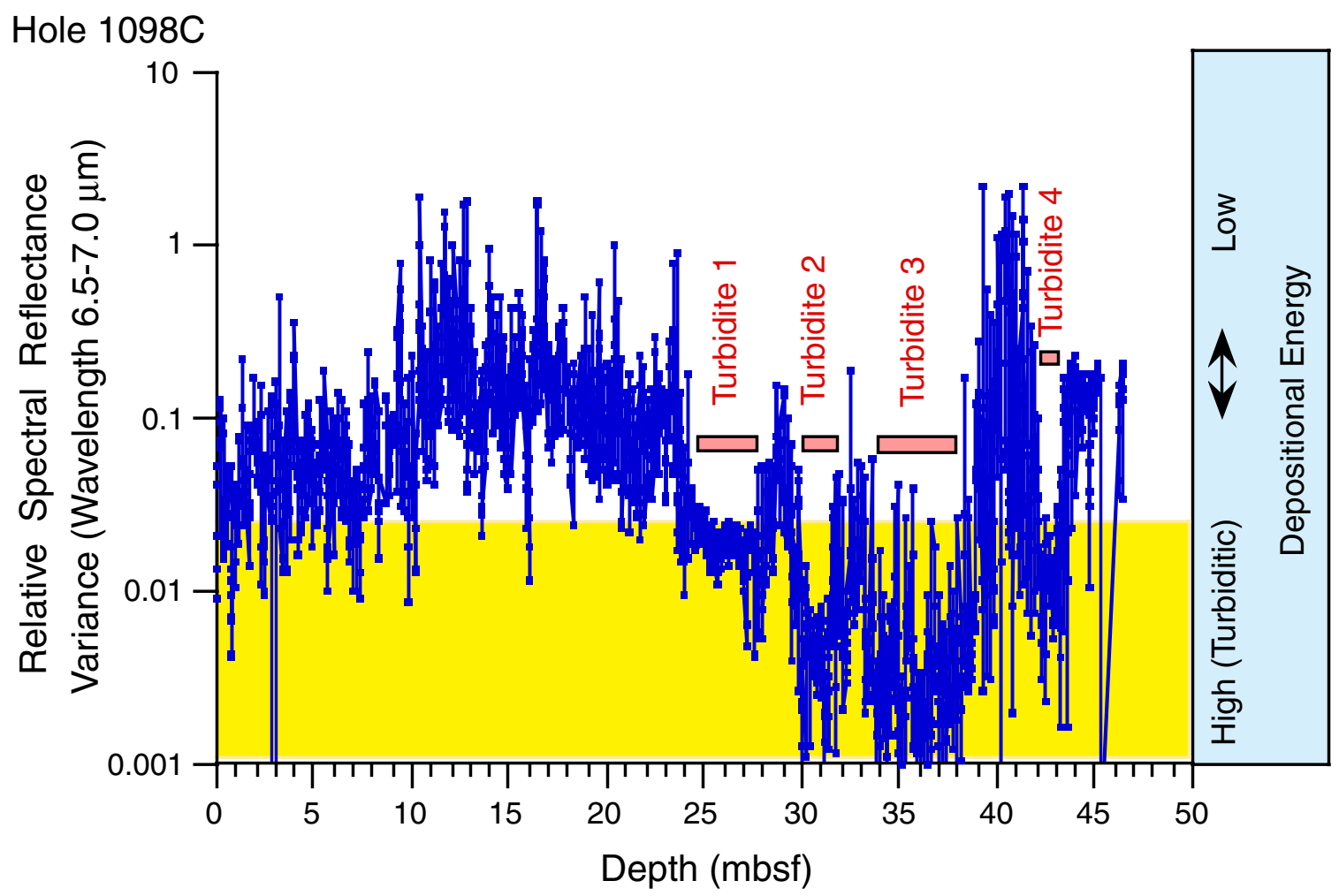


Table T1. Spectral reflectance data, Hole 1098C.

\begin{tabular}{|c|c|c|c|c|c|c|c|c|c|c|c|c|c|c|c|c|c|c|c|c|c|c|}
\hline Core & $\begin{array}{l}\text { Core } \\
\text { type }\end{array}$ & Section & $\begin{array}{l}\text { Interval } \\
\text { top } \\
(\mathrm{cm})\end{array}$ & $\begin{array}{c}\text { Interval } \\
\text { bottom } \\
(\mathrm{cm})\end{array}$ & $\begin{array}{l}\text { Curator } \\
\text { depth } \\
\text { (mbsf) }\end{array}$ & $\begin{array}{l}\text { Recovery- } \\
\text { corrected } \\
\text { depth } \\
\text { (mbsf) }\end{array}$ & $\mathrm{L}^{*}$ & $a^{*}$ & $b^{*}$ & Munsell & Munsell & Munsell & 400 & 410 & 420 & 430 & 440 & 450 & 460 & 470 & 480 & 490 \\
\hline 1 & H & 1 & 1 & 1.1 & 0.01 & 0.01 & 38.37 & 0.30 & 7.46 & $9.1 \mathrm{Y}$ & $3.7 /$ & 1.2 & 6.70 & 6.60 & 6.65 & 6.87 & 7.17 & 7.53 & 7.94 & 8.38 & 8.78 & 9.12 \\
\hline 1 & H & 1 & 3 & 3.1 & 0.03 & 0.03 & 39.16 & 0.57 & 8.22 & $8.6 \mathrm{Y}$ & $3.8 /$ & 1.3 & 6.78 & 6.72 & 6.76 & 6.97 & 7.28 & 7.69 & 8.11 & 8.54 & 8.97 & 9.36 \\
\hline 1 & $\mathrm{H}$ & 1 & 5 & 5.1 & 0.05 & 0.05 & 39.53 & 0.71 & 9.37 & $8.5 Y$ & $3.8 /$ & 1.4 & 6.41 & 6.34 & 6.41 & 6.64 & 7.00 & 7.45 & 7.93 & 8.46 & 8.97 & 9.42 \\
\hline 1 & H & 1 & 7 & 7.1 & 0.07 & 0.07 & 41.95 & 1.16 & 11.14 & $7.9 \mathrm{Y}$ & $4.0 /$ & 1.6 & 6.73 & 6.68 & 6.75 & 7.02 & 7.47 & 8.01 & 8.60 & 9.23 & 9.84 & 10.42 \\
\hline 1 & $\mathrm{H}$ & 1 & 9 & 9.1 & 0.09 & 0.09 & 43.76 & 1.42 & 12.00 & $7.5 \mathrm{Y}$ & 4.21 & 1.7 & 7.29 & 7.20 & 7.28 & 7.57 & 8.03 & 8.60 & 9.25 & 9.94 & 10.62 & 11.23 \\
\hline 1 & $\mathrm{H}$ & 1 & 11 & 11.1 & 0.11 & 0.11 & 45.68 & 1.34 & 12.22 & $7.6 \mathrm{Y}$ & $4.4 /$ & 1.8 & 8.08 & 8.00 & 8.09 & 8.41 & 8.93 & 9.55 & 10.23 & 10.97 & 11.70 & 12.37 \\
\hline 1 & H & 1 & 13 & 13.1 & 0.13 & 0.13 & 44.62 & 1.50 & 13.02 & $7.5 \mathrm{Y}$ & $4.3 /$ & 1.9 & 7.27 & 7.16 & 7.23 & 7.55 & 8.08 & 8.71 & 9.38 & 10.12 & 10.86 & 11.52 \\
\hline 1 & $\mathrm{H}$ & 1 & 15 & 15.1 & 0.15 & 0.15 & 46.01 & 1.43 & 11.39 & $7.0 \mathrm{Y}$ & $4.4 /$ & 1.6 & 8.76 & 8.68 & 8.76 & 9.06 & 9.53 & 10.12 & 10.73 & 11.38 & 12.03 & 12.64 \\
\hline 1 & $\mathrm{H}$ & 1 & 17 & 17.1 & 0.17 & 0.17 & 42.54 & 1.06 & 11.10 & $7.9 \mathrm{Y}$ & $4.1 /$ & 1.6 & 7.06 & 7.00 & 7.07 & 7.38 & 7.81 & 8.32 & 8.91 & 9.53 & 10.13 & 10.69 \\
\hline 1 & $\mathrm{H}$ & 1 & 19 & 19.1 & 0.19 & 0.19 & 43.35 & 1.51 & 12.76 & $7.2 Y$ & 4.21 & 1.8 & 6.95 & 6.87 & 6.94 & 7.23 & 7.67 & 8.22 & 8.81 & 9.43 & 10.06 & 10.66 \\
\hline 1 & $\mathrm{H}$ & 1 & 21 & 21.1 & 0.21 & 0.21 & 39.18 & 0.36 & 7.58 & $9.0 \mathrm{Y}$ & $3.8 /$ & 1.2 & 6.94 & 6.86 & 6.94 & 7.17 & 7.50 & 7.90 & 8.32 & 8.75 & 9.16 & 9.54 \\
\hline 1 & $\mathrm{H}$ & 1 & 23 & 23.1 & 0.23 & 0.23 & 43.67 & 1.16 & 10.83 & $7.6 \mathrm{Y}$ & 4.21 & 1.6 & 7.80 & 7.73 & 7.81 & 8.08 & 8.51 & 9.05 & 9.61 & 10.20 & 10.78 & 11.32 \\
\hline 1 & $\mathrm{H}$ & 1 & 25 & 25.1 & 0.25 & 0.25 & 45.90 & 1.11 & 11.52 & $7.8 \mathrm{Y}$ & $4.4 /$ & 1.7 & 8.60 & 8.50 & 8.56 & 8.86 & 9.35 & 9.95 & 10.61 & 11.30 & 11.99 & 12.64 \\
\hline 1 & H & 1 & 27 & 27.1 & 0.27 & 0.27 & 43.59 & 1.40 & 11.91 & $7.3 Y$ & 4.21 & 1.7 & 7.34 & 7.30 & 7.38 & 7.65 & 8.09 & 8.63 & 9.20 & 9.80 & 10.42 & 11.02 \\
\hline
\end{tabular}

\begin{tabular}{|c|c|c|c|c|c|c|c|c|c|c|c|c|c|c|c|c|c|c|c|c|c|}
\hline 500 & 510 & 520 & 530 & 540 & 550 & 560 & 570 & 580 & 590 & 600 & 610 & 620 & 630 & 640 & 650 & 660 & 670 & 680 & 690 & 700 & $\begin{array}{l}\text { variance }(700- \\
650 \mathrm{~nm})\end{array}$ \\
\hline 9.43 & 9. & 9.88 & .0 & 1 & .27 & 3 & 10.45 & 10.53 & 10.52 & 105 & 1049 & 10.49 & 10.54 & 10.52 & 8 & 10.44 & 10.43 & 10 & 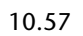 & 10.68 & 99 \\
\hline 9.71 & 10.03 & 10.21 & 1042 & 10.58 & .68 & 83 & 1 & 0 & 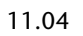 & 1 & .02 & .03 & 10 & 29 & 11.08 & .03 & 9 & 11.05 & .15 & .31 & 96667 \\
\hline 9.80 & 10.13 & 10.35 & 10.57 & 10.75 & 10.89 & 11.01 & 11.14 & 11.27 & 11.31 & 11.27 & 11.26 & 11.29 & 11.38 & 11.41 & 11.37 & 11.31 & 11.30 & 1.40 & 11.54 & 11.67 & 5667 \\
\hline 10.89 & 11.31 & 11.62 & 11.88 & 12.10 & 12.32 & 12.52 & 12.67 & 12.84 & 12.92 & 12.92 & 2.94 & 13.01 & 13.11 & 13.11 & 13.10 & 13.04 & 8 & 13.08 & 13.29 & 13.52 & 667 \\
\hline 11.77 & 12.25 & 12.64 & 12.96 & 13.24 & 13.48 & 13.71 & 13.91 & 14.11 & 14.19 & 14.22 & 4.25 & 14.32 & 14.44 & 14.49 & 14.46 & 14.34 & 14.26 & 14.38 & 14.62 & 14.89 & 0.0 \\
\hline 12.94 & 13.46 & 13.89 & 14.29 & 14.60 & 14.87 & 15.10 & 15.32 & 15.51 & 15.60 & 15.58 & 15.62 & 15.73 & 15.85 & 15.87 & 15.83 & 15.66 & 15.53 & 15.66 & 15.96 & 16.28 & 0.07332 \\
\hline 12.10 & 12.62 & 13.05 & 13.50 & 13.80 & 4.09 & 36 & 14.60 & 14.77 & 14.86 & 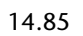 & 9 & .99 & 15 & & 15.10 & 14.89 & 14 & 14 & 15 & 15.59 & 667 \\
\hline 13.16 & 13.66 & 14.05 & 14.46 & 14.81 & 15.10 & 15.37 & 15.58 & 15.78 & 15.87 & 15.89 & 15.90 & 15.96 & 16.08 & 16.09 & 16.03 & 15.76 & 15.55 & 15.73 & 16.12 & 16.49 & 0.114266667 \\
\hline 11.15 & 11.56 & 11.93 & 12.30 & 12.53 & 12.75 & 12.94 & 13.10 & 13.23 & 13.30 & 13.29 & 13.31 & 13.37 & 13.45 & 13.49 & 13.41 & 13.22 & 13.10 & 13.25 & 13.54 & 13.81 & 0.066376667 \\
\hline 11.19 & 11.70 & 12.16 & 12.61 & 12.96 & 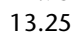 & 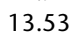 & 13. & 13. & & & 0 & 6 & & & & & & & & & \\
\hline 9.84 & 10.09 & 10.30 & 10.50 & 10.64 & 10.75 & 10.86 & 10.92 & 10.96 & 10.99 & 10.98 & 10.98 & 11.00 & 11. & 11.03 & 11.01 & 10.94 & 8 & 10.95 & 11.11 & 11.28 & 6667 \\
\hline 11.80 & 12.25 & 12.63 & 13.00 & 13.25 & 13.49 & 13.71 & 13.89 & 14.03 & 14.08 & 14.08 & 14.10 & 14.16 & 14.25 & 14.27 & 14.25 & 14.10 & 13.98 & 14.09 & 14.39 & 14.69 & 0.06684 \\
\hline 13.18 & 13.69 & 14.11 & 14.50 & 14.81 & 15.09 & 15.32 & 15.49 & 15.67 & 15.73 & 15. & 15.71 & 15.78 & 15 & & & & & 15 & 15 & 16.22 & \\
\hline 11.52 & 12.00 & 12.43 & 12.82 & 13.12 & 13.41 & 13.67 & 13.86 & 14.03 & 14.12 & 14.11 & 4.11 & 14.16 & 14.30 & 14.33 & 14.23 & 14.00 & 13.85 & 14.02 & 14.38 & 14.71 & 0.097656667 \\
\hline
\end{tabular}

Note: Only a portion of this table appears here. The complete table is available in ASCII format. 
Table T2. Spectral reflectance data, Hole 1095A.

\begin{tabular}{|c|c|c|c|c|c|c|c|c|c|c|c|c|c|c|c|c|c|c|c|c|c|}
\hline Core & $\begin{array}{l}\text { Core } \\
\text { type }\end{array}$ & Section & $\begin{array}{l}\text { Interval } \\
\text { top } \\
(\mathrm{cm})\end{array}$ & $\begin{array}{c}\text { Interval } \\
\text { bottom } \\
(\mathrm{cm})\end{array}$ & $\begin{array}{c}\text { Curator } \\
\text { depth } \\
\text { (mbsf) }\end{array}$ & $\begin{array}{l}\text { Recovery- } \\
\text { corrected } \\
\text { depth } \\
\text { (mbsf) }\end{array}$ & $\mathrm{L}^{*}$ & $a^{*}$ & $b^{*}$ & Munsell & Munsell & Munsell & 400 & 410 & 420 & 430 & 440 & 450 & 460 & 470 & 480 \\
\hline 1 & $\mathrm{H}$ & 1 & 2 & 2.1 & 0.02 & 0.02 & 96.73 & -0.29 & -0.28 & $0.1 \mathrm{~PB}$ & $9.6 /$ & 0.1 & 92.86 & 92.66 & 92.40 & 92.32 & 92.20 & 92.22 & 92.12 & 92.14 & 92.12 \\
\hline 1 & $\mathrm{H}$ & 1 & 7 & 7.1 & 0.07 & 0.07 & 96.74 & -0.29 & -0.28 & $9.8 \mathrm{~B}$ & $9.6 /$ & 0.1 & 92.86 & 92.65 & 92.41 & 92.35 & 92.23 & 92.20 & 92.11 & 92.14 & 92.13 \\
\hline 1 & $\mathrm{H}$ & 1 & 2 & 2.1 & 0.02 & 0.02 & 37.75 & 2.15 & 5.07 & $1.6 \mathrm{Y}$ & $3.6 /$ & 0.7 & 7.48 & 7.60 & 7.77 & 7.95 & 8.13 & 8.28 & 8.38 & 8.51 & 8.64 \\
\hline 1 & $\mathrm{H}$ & 1 & 7 & 7.1 & 0.07 & 0.07 & 40.77 & 2.88 & 8.70 & $2.8 \mathrm{Y}$ & $3.9 /$ & 1.2 & 7.00 & 7.24 & 7.54 & 7.91 & 8.28 & 8.59 & 8.85 & 9.12 & 9.35 \\
\hline 1 & $\mathrm{H}$ & 1 & 12 & 12.1 & 0.12 & 0.12 & 41.66 & 2.91 & 9.62 & $3.3 Y$ & $4.0 /$ & 1.3 & 6.85 & 7.17 & 7.51 & 7.92 & 8.36 & 8.75 & 9.05 & 9.33 & 9.61 \\
\hline 1 & $\mathrm{H}$ & 1 & 17 & 17.1 & 0.17 & 0.17 & 41.26 & 1.53 & 6.30 & $4.3 Y$ & $4.0 /$ & 0.9 & 8.08 & 8.34 & 8.65 & 9.01 & 9.37 & 9.67 & 9.89 & 10.08 & 10.29 \\
\hline 1 & $\mathrm{H}$ & 1 & 22 & 22.1 & 0.22 & 0.22 & 35.58 & 1.65 & 5.91 & $3.9 \mathrm{Y}$ & $3.4 /$ & 0.8 & 5.81 & 6.04 & 6.27 & 6.53 & 6.80 & 7.02 & 7.16 & 7.30 & 7.45 \\
\hline 1 & $\mathrm{H}$ & 1 & 27 & 27.1 & 0.27 & 0.27 & 26.64 & 1.18 & 4.01 & $4.0 \mathrm{Y}$ & $2.6 /$ & 0.6 & 3.52 & 3.61 & 3.75 & 3.91 & 4.04 & 4.13 & 4.20 & 4.28 & 4.35 \\
\hline 1 & $\mathrm{H}$ & 1 & 32 & 32.1 & 0.32 & 0.32 & 32.48 & 1.50 & 5.33 & $4.0 \mathrm{Y}$ & $3.1 /$ & 0.8 & 4.91 & 5.08 & 5.29 & 5.51 & 5.72 & 5.88 & 6.00 & 6.11 & 6.22 \\
\hline 1 & $\mathrm{H}$ & 1 & 37 & 37.1 & 0.37 & 0.37 & 35.60 & 2.37 & 7.93 & $3.6 \mathrm{Y}$ & $3.4 /$ & 1.1 & 4.99 & 5.27 & 5.55 & 5.87 & 6.19 & 6.46 & 6.67 & 6.85 & 7.03 \\
\hline 1 & $\mathrm{H}$ & 1 & 42 & 42.1 & 0.42 & 0.42 & 39.41 & 2.17 & 7.88 & $3.8 \mathrm{Y}$ & $3.8 /$ & 1.1 & 6.37 & 6.69 & 7.03 & 7.45 & 7.86 & 8.20 & 8.46 & 8.67 & 8.87 \\
\hline 1 & $\mathrm{H}$ & 1 & 47 & 47.1 & 0.47 & 0.47 & 41.55 & 2.12 & 7.44 & $3.6 \mathrm{Y}$ & $4.0 /$ & 1 & 7.67 & 7.95 & 8.28 & 8.70 & 9.11 & 9.44 & 9.69 & 9.92 & 10.14 \\
\hline 1 & $\mathrm{H}$ & 1 & 52 & 52.1 & 0.52 & 0.52 & 44.72 & 2.35 & 8.67 & $3.7 Y$ & $4.3 /$ & 1.2 & 8.56 & 8.93 & 9.34 & 9.84 & 10.36 & 10.79 & 11.10 & 11.37 & 11.63 \\
\hline 1 & $\mathrm{H}$ & 1 & 57 & 57.1 & 0.57 & 0.57 & 47.25 & 2.54 & 9.24 & $3.6 \mathrm{Y}$ & $4.5 /$ & 1.3 & 9.47 & 9.95 & 10.43 & 10.98 & 11.57 & 12.10 & 12.48 & 12.81 & 13.13 \\
\hline
\end{tabular}

\begin{tabular}{|c|c|c|c|c|c|c|c|c|c|c|c|c|c|c|c|c|c|c|c|c|c|}
\hline 490 & 500 & 510 & 520 & 530 & 540 & 550 & 560 & 570 & 580 & 590 & 600 & 610 & 620 & 630 & 640 & 650 & 660 & 670 & 680 & 690 & 700 \\
\hline 92.18 & 92.14 & 92.13 & 92.07 & 92.05 & 91.95 & 91.95 & 91.92 & 91.81 & 91.79 & 91.75 & 91.66 & 91.55 & 91.38 & 91.40 & 91.24 & 91.25 & 91.21 & 91.19 & 91.16 & 91.16 & 91.19 \\
\hline 92.17 & 92.14 & 92.15 & 92.09 & 92.06 & 91.96 & 91.93 & 91.91 & 91.85 & 91.82 & 91.73 & 91.61 & 91.55 & 91.41 & 91.41 & 91.20 & 91.23 & 91.24 & 91.15 & 91.15 & 91.20 & 91.17 \\
\hline 8.75 & 8.87 & 9.04 & 9.20 & 9.39 & 9.55 & 9.70 & 9.84 & 9.98 & 10.14 & 10.25 & 10.34 & 10.43 & 10.52 & 10.62 & 10.70 & 10.80 & 10.87 & 10.91 & 10.99 & 11.03 & 11.05 \\
\hline 9.56 & 9.81 & 10.15 & 10.46 & 10.77 & 11.08 & 11.37 & 11.61 & 11.88 & 12.10 & 12.27 & 12.37 & 12.48 & 12.61 & 12.70 & 12.80 & 12.92 & 13.00 & 13.03 & 13.07 & 13.12 & 13.17 \\
\hline 9.88 & 10.17 & 10.53 & 10.89 & 11.28 & 11.60 & 11.91 & 12.19 & 12.48 & 12.70 & 12.89 & 13.01 & 13.11 & 13.22 & 13.31 & 13.37 & 13.45 & 13.54 & 13.59 & 13.64 & 13.69 & 13.76 \\
\hline 10.48 & 10.68 & 10.93 & 11.19 & 11.43 & 11.65 & 11.88 & 12.06 & 12.17 & 12.32 & 12.41 & 12.47 & 12.49 & 12.53 & 12.54 & 12.56 & 12.62 & 12.64 & 12.62 & 12.64 & 12.66 & 12.66 \\
\hline 7.60 & 7.74 & 7.93 & 8.13 & 8.31 & 8.46 & 8.62 & 8.77 & 8.91 & 9.03 & 9.10 & 9.15 & 9.18 & 9.21 & 9.26 & 9.27 & 9.30 & 9.33 & 9.34 & 9.38 & 9.38 & 9.40 \\
\hline 4.41 & 4.47 & 4.56 & 4.65 & 4.75 & 4.80 & 4.86 & 4.95 & 5.02 & 5.08 & 5.15 & 5.14 & 5.15 & 5.17 & 5.19 & 5.21 & 5.20 & 5.20 & 5.23 & 5.24 & 5.24 & 5.26 \\
\hline 6.33 & 6.46 & 6.62 & 6.78 & 6.91 & 7.03 & 7.17 & 7.26 & 7.41 & 7.49 & 7.56 & 7.58 & 7.61 & 7.64 & 7.67 & 7.68 & 7.70 & 7.73 & 7.74 & 7.76 & 7.76 & 7.77 \\
\hline 7.21 & 7.42 & 7.65 & 7.88 & 8.12 & 8.34 & 8.54 & 8.74 & 8.94 & 9.11 & 9.25 & 9.31 & 9.35 & 9.43 & 9.45 & 9.48 & 9.53 & 9.54 & 9.56 & 9.59 & 9.63 & 9.66 \\
\hline 9.10 & 9.35 & 9.61 & 9.90 & 10.16 & 10.39 & 10.63 & 10.88 & 11.06 & 11.26 & 11.38 & 11.46 & 11.51 & 11.55 & 11.60 & 11.61 & 11.65 & 11.67 & 11.70 & 11.74 & 11.77 & 11.80 \\
\hline 10.36 & 10.58 & 10.86 & 11.14 & 11.44 & 11.70 & 11.94 & 12.18 & 12.37 & 12.57 & 12.75 & 12.80 & 12.83 & 12.89 & 12.94 & 12.96 & 12.99 & 13.05 & 13.07 & 13.09 & 13.13 & 13.16 \\
\hline 11.93 & 12.24 & 12.61 & 12.97 & 13.35 & 13.72 & 14.03 & 14.32 & 14.59 & 14.82 & 14.98 & 15.04 & 15.09 & 15.18 & 15.26 & 15.28 & 15.32 & 15.40 & 15.46 & 15.51 & 15.55 & 15.62 \\
\hline 13.46 & 13.81 & 14.23 & 14.66 & 15.07 & 15.44 & 15.82 & 16.18 & 16.47 & 16.76 & 16.96 & 17.05 & 17.14 & 17.20 & 17.26 & 17.30 & 17.37 & 17.41 & 17.45 & 17.53 & 17.56 & 17.59 \\
\hline
\end{tabular}

Note: Only a portion of this table appears here. The complete table is available in ASCII format. 
Table T3. Spectral reflectance data, Hole 1095B.

\begin{tabular}{|c|c|c|c|c|c|c|c|c|c|c|c|c|c|c|c|c|c|c|c|c|c|}
\hline Core & $\begin{array}{l}\text { Core } \\
\text { type }\end{array}$ & Section & $\begin{array}{l}\text { Interval } \\
\text { top } \\
(\mathrm{cm})\end{array}$ & $\begin{array}{l}\text { Interval } \\
\text { bottom } \\
(\mathrm{cm})\end{array}$ & $\begin{array}{l}\text { Curator } \\
\text { depth } \\
\text { (mbsf) }\end{array}$ & $\begin{array}{l}\text { Recovery- } \\
\text { corrected } \\
\text { depth } \\
\text { (mbsf) }\end{array}$ & $L^{*}$ & $a^{*}$ & $b^{*}$ & Munsell & Munsell & Munsell & 400 & 410 & 420 & 430 & 440 & 450 & 460 & 470 & 480 \\
\hline 1 & $\mathrm{H}$ & 1 & 2 & 2.1 & 83.02 & 83.02 & 27.42 & -0.51 & 0.28 & $0.3 G$ & $2.7 /$ & 0.2 & 5.08 & 5.10 & 5.11 & 5.13 & 5.13 & 5.15 & 5.18 & 5.22 & 5.23 \\
\hline 1 & $\mathrm{H}$ & 1 & 7 & 7.1 & 83.07 & 83.07 & 49.17 & -1.69 & 2.90 & $6.0 \mathrm{GY}$ & $4.8 /$ & 0.6 & 14.28 & 14.61 & 15.01 & 15.44 & 15.79 & 16.07 & 16.35 & 16.67 & 16.99 \\
\hline 1 & $\mathrm{H}$ & 1 & 12 & 12.1 & 83.12 & 83.12 & 48.79 & -2.18 & 1.96 & $8.8 \mathrm{GY}$ & $4.7 /$ & 0.6 & 14.91 & 15.16 & 15.42 & 15.74 & 15.99 & 16.23 & 16.47 & 16.75 & 17.04 \\
\hline 1 & $\mathrm{H}$ & 1 & 17 & 17.1 & 83.17 & 83.16 & 26.42 & -0.70 & 1.01 & $6.2 \mathrm{GY}$ & $2.6 /$ & 0.3 & 4.27 & 4.38 & 4.47 & 4.55 & 4.62 & 4.64 & 4.67 & 4.72 & 4.78 \\
\hline 1 & $\mathrm{H}$ & 1 & 22 & 22.1 & 83.22 & 83.21 & 39.25 & -0.18 & 1.58 & $0.8 \mathrm{GY}$ & $3.8 /$ & 0.3 & 9.85 & 9.84 & 9.92 & 10.04 & 10.11 & 10.16 & 10.25 & 10.36 & 10.43 \\
\hline 1 & $\mathrm{H}$ & 1 & 27 & 27.1 & 83.27 & 83.26 & 34.81 & -0.60 & 1.43 & $5.0 \mathrm{GY}$ & $3.4 /$ & 0.3 & 7.09 & 7.29 & 7.54 & 7.76 & 7.87 & 7.91 & 7.97 & 8.07 & 8.16 \\
\hline 1 & $\mathrm{H}$ & 1 & 32 & 32.1 & 83.32 & 83.31 & 39.08 & -0.77 & 0.78 & $7.7 \mathrm{GY}$ & $3.8 /$ & 0.2 & 10.07 & 10.11 & 10.18 & 10.27 & 10.32 & 10.37 & 10.41 & 10.48 & 10.57 \\
\hline 1 & $\mathrm{H}$ & 1 & 37 & 37.1 & 83.37 & 83.36 & 44.49 & -1.00 & 1.10 & $7.5 \mathrm{GY}$ & $4.3 /$ & 0.3 & 12.96 & 13.08 & 13.21 & 13.39 & 13.52 & 13.62 & 13.71 & 13.82 & 13.94 \\
\hline 1 & $\mathrm{H}$ & 1 & 42 & 42.1 & 83.42 & 83.41 & 31.15 & -0.79 & 1.36 & $5.7 \mathrm{GY}$ & $3.0 /$ & 0.4 & 5.91 & 5.98 & 6.06 & 6.17 & 6.26 & 6.31 & 6.35 & 6.44 & 6.52 \\
\hline 1 & $\mathrm{H}$ & 1 & 47 & 47.1 & 83.47 & 83.45 & 36.37 & -0.80 & 0.73 & $8.2 \mathrm{GY}$ & $3.5 /$ & 0.2 & 8.64 & 8.66 & 8.73 & 8.82 & 8.88 & 8.91 & 8.95 & 9.03 & 9.11 \\
\hline 1 & $\mathrm{H}$ & 1 & 52 & 52.1 & 83.52 & 83.50 & 38.57 & -0.79 & 0.54 & $10.0 \mathrm{GY}$ & $3.8 /$ & 0.2 & 9.81 & 9.88 & 9.97 & 10.08 & 10.13 & 10.17 & 10.21 & 10.28 & 10.34 \\
\hline 1 & $\mathrm{H}$ & 1 & 57 & 57.1 & 83.57 & 83.55 & 35.44 & -0.59 & 0.50 & $8.6 \mathrm{GY}$ & $3.5 /$ & 0.2 & 8.26 & 8.31 & 8.39 & 8.45 & 8.49 & 8.53 & 8.56 & 8.61 & 8.66 \\
\hline 1 & $\mathrm{H}$ & 1 & 62 & 62.1 & 83.62 & 83.60 & 34.31 & -0.49 & 0.65 & $6.5 \mathrm{GY}$ & $3.3 /$ & 0.2 & 7.77 & 7.76 & 7.80 & 7.86 & 7.90 & 7.93 & 7.95 & 8.01 & 8.06 \\
\hline 1 & $\mathrm{H}$ & 1 & 67 & 67.1 & 83.67 & 83.65 & 46.89 & -0.16 & 3.20 & $9.8 Y$ & $4.5 /$ & 0.5 & 13.31 & 13.44 & 13.67 & 13.94 & 14.15 & 14.34 & 14.52 & 14.72 & 14.93 \\
\hline
\end{tabular}

\begin{tabular}{|c|c|c|c|c|c|c|c|c|c|c|c|c|c|c|c|c|c|c|c|c|c|}
\hline 490 & 500 & 510 & 520 & 530 & 540 & 550 & 560 & 570 & 580 & 590 & 600 & 610 & 620 & 630 & 640 & 650 & 660 & 670 & 680 & 690 & 700 \\
\hline 5.24 & 5.27 & 5.30 & 5.29 & 5.30 & 5.28 & 5.27 & 5.30 & 5.29 & 5.25 & 5.24 & 5.22 & 5.19 & 5.18 & 5.15 & 5.12 & 5.09 & 5.08 & 5.05 & 5.03 & 5.02 & 5.01 \\
\hline 17.28 & 17.50 & 17.71 & 17.88 & 17.97 & 18.02 & 18.10 & 18.10 & 18.07 & 18.01 & 17.87 & 17.67 & 17.45 & 17.21 & 17.03 & 16.79 & 16.54 & 16.28 & 16.02 & 15.81 & 15.60 & 15.38 \\
\hline 17.30 & 17.49 & 17.68 & 17.81 & 17.85 & 17.87 & 17.88 & 17.83 & 17.73 & 17.61 & 17.46 & 17.23 & 16.98 & 16.76 & 16.53 & 16.25 & 15.98 & 15.71 & 15.45 & 15.21 & 14.96 & 14.75 \\
\hline 4.84 & 4.87 & 4.91 & 4.92 & 4.92 & 4.95 & 4.99 & 4.95 & 4.93 & 4.93 & 4.90 & 4.87 & 4.84 & 4.80 & 4.79 & 4.74 & 4.67 & 4.62 & 4.59 & 4.56 & 4.54 & 4.50 \\
\hline 10.51 & 10.57 & 10.66 & 10.77 & 10.79 & 10.81 & 10.83 & 10.88 & 10.87 & 10.87 & 10.86 & 10.82 & 10.79 & 10.80 & 10.81 & 10.75 & 10.69 & 10.66 & 10.64 & 10.62 & 10.58 & 10.55 \\
\hline 8.25 & 8.33 & 8.38 & 8.41 & 8.44 & 8.47 & 8.48 & 8.50 & 8.49 & 8.47 & 8.44 & 8.38 & 8.35 & 8.31 & 8.27 & 8.21 & 8.16 & 8.10 & 8.02 & 7.96 & 7.92 & 7.90 \\
\hline 10.64 & 10.69 & 10.74 & 10.78 & 10.82 & 10.81 & 10.82 & 10.84 & 10.79 & 10.76 & 10.71 & 10.67 & 10.59 & 10.52 & 10.46 & 10.40 & 10.32 & 10.26 & 10.21 & 10.13 & 10.04 & 9.99 \\
\hline 14.07 & 14.11 & 14.20 & 14.33 & 14.35 & 14.35 & 14.36 & 14.37 & 14.32 & 14.27 & 14.19 & 14.10 & 13.99 & 13.91 & 13.83 & 13.71 & 13.60 & 13.48 & 13.36 & 13.27 & 13.17 & 13.09 \\
\hline 6.58 & 6.62 & 6.69 & 6.77 & 6.77 & 6.79 & 6.81 & 6.81 & 6.78 & 6.76 & 6.76 & 6.71 & 6.64 & 6.61 & 6.53 & 6.45 & 6.42 & 6.37 & 6.31 & 6.23 & 6.18 & 6.16 \\
\hline 9.15 & 9.18 & 9.25 & 9.30 & 9.33 & 9.31 & 9.30 & 9.30 & 9.28 & 9.27 & 9.22 & 9.15 & 9.09 & 9.04 & 9.00 & 8.91 & 8.83 & 8.78 & 8.73 & 8.67 & 8.60 & 8.58 \\
\hline 10.39 & 10.42 & 10.46 & 10.53 & 10.54 & 10.52 & 10.54 & 10.53 & 10.48 & 10.43 & 10.39 & 10.35 & 10.29 & 10.25 & 10.19 & 10.09 & 10.02 & 9.98 & 9.93 & 9.86 & 9.78 & 9.75 \\
\hline 8.71 & 8.74 & 8.77 & 8.77 & 8.78 & 8.80 & 8.80 & 8.80 & 8.78 & 8.77 & 8.72 & 8.66 & 8.65 & 8.64 & 8.57 & 8.51 & 8.47 & 8.41 & 8.36 & 8.35 & 8.30 & 8.24 \\
\hline 8.09 & 8.11 & 8.17 & 8.19 & 8.22 & 8.23 & 8.22 & 8.21 & 8.21 & 8.19 & 8.18 & 8.12 & 8.11 & 8.07 & 8.04 & 8.00 & 7.97 & 7.90 & 7.86 & 7.87 & 7.85 & 7.80 \\
\hline 15.13 & 15.30 & 15.51 & 15.66 & 15.78 & 15.88 & 15.97 & 16.08 & 16.15 & 16.15 & 16.15 & 16.10 & 16.03 & 15.93 & 15.87 & 15.77 & 15.70 & 15.61 & 15.47 & 15.37 & 15.28 & 15.21 \\
\hline
\end{tabular}

Note: Only a portion of this table appears here. The complete table is available in ASCII format. 
Table T4. Spectral reflectance data, Hole 1095C.

\begin{tabular}{|c|c|c|c|c|c|c|c|c|c|c|c|c|c|c|c|c|c|c|c|c|c|}
\hline Core & $\begin{array}{l}\text { Core } \\
\text { type }\end{array}$ & Section & $\begin{array}{l}\text { Interval } \\
\text { top } \\
(\mathrm{cm})\end{array}$ & $\begin{array}{l}\text { Interval } \\
\text { bottom } \\
(\mathrm{cm})\end{array}$ & $\begin{array}{l}\text { Curator } \\
\text { depth } \\
\text { (mbsf) }\end{array}$ & $\begin{array}{l}\text { Recovery- } \\
\text { corrected } \\
\text { depth } \\
\text { (mbsf) }\end{array}$ & $L^{*}$ & $a^{*}$ & $b^{*}$ & Munsell & Munsell & Munsell & 400 & 410 & 420 & 430 & 440 & 450 & 460 & 470 & 480 \\
\hline 1 & $\mathrm{H}$ & 1 & 2 & 2.1 & 0.02 & 0.02 & 49.70 & 4.03 & 11.96 & $2.5 \mathrm{Y}$ & $4.7 /$ & 1.7 & 10.02 & 10.39 & 10.80 & 11.34 & 11.94 & 12.51 & 13.00 & 13.41 & 13.81 \\
\hline 1 & $\mathrm{H}$ & 1 & 7 & 7.1 & 0.07 & 0.07 & 45.03 & 1.21 & 5.53 & $4.5 \mathrm{Y}$ & $4.3 /$ & 0.8 & 10.41 & 10.70 & 11.04 & 11.47 & 11.87 & 12.18 & 12.42 & 12.63 & 12.83 \\
\hline 1 & $\mathrm{H}$ & 1 & 12 & 12.1 & 0.12 & 0.12 & 51.18 & 0.72 & 5.71 & $6.4 \mathrm{Y}$ & $4.9 /$ & 0.8 & 14.01 & 14.44 & 14.85 & 15.36 & 15.92 & 16.42 & 16.78 & 17.05 & 17.31 \\
\hline 1 & $\mathrm{H}$ & 1 & 17 & 17.1 & 0.17 & 0.17 & 51.81 & 3.69 & 11.68 & $2.9 \mathrm{Y}$ & $5.0 /$ & 1.6 & 11.10 & 11.60 & 12.07 & 12.67 & 13.40 & 14.07 & 14.59 & 15.04 & 15.47 \\
\hline 1 & $\mathrm{H}$ & 1 & 22 & 22.1 & 0.22 & 0.22 & 52.66 & 2.23 & 7.18 & $3.0 \mathrm{Y}$ & $5.1 /$ & 1 & 14.71 & 15.06 & 15.43 & 15.89 & 16.41 & 16.88 & 17.23 & 17.50 & 17.80 \\
\hline 1 & $\mathrm{H}$ & 1 & 27 & 27.1 & 0.27 & 0.27 & 46.80 & 2.27 & 7.15 & $3.0 \mathrm{Y}$ & $4.5 /$ & 1 & 10.85 & 11.14 & 11.49 & 11.90 & 12.29 & 12.66 & 12.96 & 13.18 & 13.41 \\
\hline 1 & $\mathrm{H}$ & 1 & 32 & 32.1 & 0.32 & 0.32 & 37.95 & 1.01 & 3.41 & $3.5 \mathrm{Y}$ & $3.7 /$ & 0.5 & 8.22 & 8.32 & 8.45 & 8.61 & 8.77 & 8.91 & 9.00 & 9.09 & 9.16 \\
\hline 1 & $\mathrm{H}$ & 1 & 37 & 37.1 & 0.37 & 0.37 & 39.98 & 1.45 & 4.72 & $3.3 Y$ & $3.9 /$ & 0.7 & 8.50 & 8.67 & 8.85 & 9.08 & 9.33 & 9.53 & 9.68 & 9.81 & 9.94 \\
\hline 1 & $\mathrm{H}$ & 1 & 42 & 42.1 & 0.42 & 0.42 & 44.75 & 1.82 & 5.87 & $3.1 \mathrm{Y}$ & $4.3 /$ & 0.8 & 10.30 & 10.55 & 10.86 & 11.25 & 11.61 & 11.89 & 12.11 & 12.29 & 12.47 \\
\hline 1 & $\mathrm{H}$ & 1 & 47 & 47.1 & 0.47 & 0.47 & 49.90 & 1.27 & 4.16 & $3.2 Y$ & $4.8 /$ & 0.6 & 14.74 & 15.00 & 15.32 & 15.66 & 15.97 & 16.24 & 16.42 & 16.57 & 16.73 \\
\hline 1 & $\mathrm{H}$ & 1 & 52 & 52.1 & 0.52 & 0.52 & 53.42 & 1.76 & 6.50 & $3.5 Y$ & $5.1 /$ & 0.9 & 15.53 & 15.94 & 16.36 & 16.88 & 17.39 & 17.84 & 18.16 & 18.44 & 18.73 \\
\hline 1 & $\mathrm{H}$ & 1 & 57 & 57.1 & 0.57 & 0.57 & 49.48 & 1.56 & 5.42 & $3.4 \mathrm{Y}$ & $4.8 /$ & 0.8 & 13.72 & 13.93 & 14.21 & 14.58 & 14.97 & 15.30 & 15.54 & 15.76 & 15.96 \\
\hline 1 & $\mathrm{H}$ & 1 & 62 & 62.1 & 0.62 & 0.62 & 53.45 & 1.89 & 6.69 & $3.4 \mathrm{Y}$ & $5.1 /$ & 0.9 & 15.52 & 15.86 & 16.24 & 16.74 & 17.30 & 17.76 & 18.09 & 18.38 & 18.69 \\
\hline 1 & $\mathrm{H}$ & 1 & 67 & 67.1 & 0.67 & 0.67 & 51.79 & 2.34 & 8.62 & $3.6 \mathrm{Y}$ & $5.0 /$ & 1.2 & 12.64 & 13.13 & 13.64 & 14.26 & 14.91 & 15.49 & 15.92 & 16.28 & 16.62 \\
\hline 1 & $\mathrm{H}$ & 1 & 72 & 72.1 & 0.72 & 0.72 & 46.79 & 1.87 & 6.56 & $3.5 \mathrm{Y}$ & $4.5 /$ & 0.9 & 11.03 & 11.34 & 11.69 & 12.11 & 12.54 & 12.91 & 13.18 & 13.41 & 13.63 \\
\hline
\end{tabular}

\begin{tabular}{|c|c|c|c|c|c|c|c|c|c|c|c|c|c|c|c|c|c|c|c|c|c|}
\hline 490 & 500 & 510 & 520 & 530 & 540 & 550 & 560 & 570 & 580 & 590 & 600 & 610 & 620 & 630 & 640 & 650 & 660 & 670 & 680 & 690 & 700 \\
\hline 13.04 & 13.26 & 13.5 & 13.72 & 13.97 & 14.19 & 14.41 & 14.63 & 14.77 & 14.89 & 14.95 & 14.98 & 15.02 & 15.01 & 15.02 & 15.00 & 15.04 & 15.07 & 5.07 & 15.06 & 15.06 & 15.07 \\
\hline 17.60 & 17.91 & 18.24 & 18.53 & 18.88 & 19.12 & 19.36 & 19.56 & 19.72 & 19.80 & 19.84 & 19.83 & 19.83 & 19.81 & 19.77 & 19.73 & 19.71 & 19.71 & 19.71 & 19.66 & 19.62 & 19.60 \\
\hline 15.94 & 16.46 & 17.05 & 17.61 & 18.2 & 18.74 & 19.30 & 19.85 & 20.30 & 20.68 & 21.00 & 21.25 & 21.43 & 21.59 & 21.77 & 21.88 & 22.06 & 22.24 & 22.35 & 22.42 & 22.50 & 22.61 \\
\hline 18.12 & 18.46 & 18.84 & 19.22 & 19.61 & 19.96 & 20.31 & 20.67 & 20.97 & 21.22 & 21.43 & 21.57 & 21.66 & 21.73 & 21.83 & 21.89 & 21.98 & 22.06 & 22.10 & 22.16 & 22.20 & 22.23 \\
\hline 13.67 & 13.97 & 14.29 & 14.58 & 14.92 & 15.23 & 15.51 & 15.78 & 16.06 & 16.25 & 16.46 & 16.55 & 16.62 & 16.70 & 16.81 & 16.85 & 16.94 & 17.03 & 17.07 & 17.10 & 17.15 & 17.18 \\
\hline 9.26 & 9.36 & 9.49 & 9.62 & 9.73 & 9.83 & 9.95 & 10.07 & 10.13 & 10.22 & 10.26 & 10.27 & 10.32 & 10.35 & 10.37 & 10.37 & 10.39 & 10.41 & 10.44 & 10.44 & 10.44 & 10.47 \\
\hline 10.09 & 10.24 & 10.42 & 10.57 & 10.74 & 10.88 & 11.06 & 11.22 & 11.36 & 11.46 & 11.54 & 11.59 & 11.64 & 11.70 & 11.71 & 11.70 & 11.74 & 11.78 & 11.79 & 11.80 & 11.84 & 11.88 \\
\hline 12.68 & 12.90 & 13.14 & 13.39 & 13.63 & 13.88 & 14.10 & 14.32 & 14.53 & 14.68 & 14.82 & 14.89 & 14.96 & 15.01 & 15.07 & 15.10 & 15.14 & 15.17 & 15.22 & 15.26 & 15.28 & 15.29 \\
\hline 16.92 & 17.12 & 17.33 & 17.49 & 17.71 & 17.93 & 18.14 & 18.30 & 18.45 & 18.61 & 18.72 & 18.76 & 18.82 & 18.85 & 18.91 & 18.92 & 18.96 & 19.01 & 19.03 & 19.05 & 19.05 & 19.08 \\
\hline 19.02 & 19.33 & 19.73 & 20.11 & 20.48 & 20.81 & 21.14 & 21.42 & 21.68 & 21.91 & 22.07 & 22.14 & 22.19 & 22.26 & 22.35 & 22.34 & 22.36 & 22.41 & 22.49 & 22.54 & 22.55 & 22.57 \\
\hline 16.18 & 16.42 & 16.71 & 16.97 & 17.22 & 17.46 & 17.73 & 17.97 & 18.18 & 18.34 & 18.47 & 18.52 & 18.55 & 18.64 & 18.68 & 18.68 & 18.78 & 18.82 & 18.82 & 18.85 & 18.88 & 18.94 \\
\hline 19.00 & 19.32 & 19.71 & 20.09 & 20.43 & 20.80 & 21.13 & 21.42 & 21.71 & 21.93 & 22.11 & 22.17 & 22.28 & 22.36 & 22.43 & 22.45 & 22.52 & 22.58 & 22.63 & 22.71 & 22.74 & 22.79 \\
\hline 16.99 & 17.38 & 17.85 & 18.28 & 18.73 & 19.14 & 19.56 & 19.93 & 20.27 & 20.55 & 20.73 & 20.85 & 20.95 & 21.00 & 21.07 & 21.10 & 21.16 & 21.26 & 21.31 & 21.33 & 21.38 & 21.45 \\
\hline 13.89 & 14.15 & 14.44 & 14.71 & 15.02 & 15.30 & 15.57 & 15.83 & 16.07 & 16.24 & 16.40 & 16.47 & 16.51 & 16.58 & 16.63 & 16.61 & 16.65 & 16.73 & 16.78 & 16.80 & 16.81 & 16.85 \\
\hline
\end{tabular}

Note: Only a portion of this table appears here. The complete table is available in ASCII format. 
Table T5. Spectral reflectance data, Hole 1095D.

\begin{tabular}{|c|c|c|c|c|c|c|c|c|c|c|c|c|c|c|c|c|c|c|c|c|c|}
\hline Core & $\begin{array}{l}\text { Core } \\
\text { type }\end{array}$ & Section & $\begin{array}{l}\text { Interval } \\
\text { top } \\
(\mathrm{cm})\end{array}$ & $\begin{array}{l}\text { Interval } \\
\text { bottom } \\
(\mathrm{cm})\end{array}$ & $\begin{array}{l}\text { Curator } \\
\text { depth } \\
\text { (mbsf) }\end{array}$ & $\begin{array}{l}\text { Recovery- } \\
\text { corrected } \\
\text { depth } \\
\text { (mbsf) }\end{array}$ & $\mathrm{L}^{*}$ & $a^{*}$ & $b^{*}$ & Munsell & Munsell & Munsell & 400 & 410 & 420 & 430 & 440 & 450 & 460 & 470 & 480 \\
\hline 1 & $\mathrm{H}$ & CC & 2 & 2.1 & 8.36 & 8.36 & 42.32 & 3.59 & 8.05 & $0.8 \mathrm{Y}$ & $4.1 /$ & 1.1 & 8.55 & 8.72 & 8.90 & 9.14 & 9.39 & 9.64 & 9.87 & 10.06 & 10.27 \\
\hline 1 & $\mathrm{H}$ & CC & 7 & 7.1 & 8.41 & 8.41 & 45.99 & 2.39 & 7.24 & $2.8 \mathrm{Y}$ & $4.4 /$ & 1 & 10.52 & 10.75 & 11.01 & 11.36 & 11.77 & 12.12 & 12.37 & 12.61 & 12.83 \\
\hline 1 & $\mathrm{H}$ & CC & 12 & 12.1 & 8.46 & 8.46 & 48.25 & 1.43 & 6.07 & $4.2 Y$ & $4.6 /$ & 0.8 & 12.15 & 12.49 & 12.84 & 13.29 & 13.74 & 14.10 & 14.39 & 14.64 & 14.86 \\
\hline 1 & $\mathrm{H}$ & CC & 17 & 17.1 & 8.51 & 8.51 & 48.53 & 1.36 & 5.27 & $3.8 \mathrm{Y}$ & $4.7 /$ & 0.7 & 13.04 & 13.31 & 13.60 & 13.97 & 14.36 & 14.67 & 14.90 & 15.12 & 15.33 \\
\hline 1 & $\mathrm{H}$ & CC & 22 & 22.1 & & & 47.51 & 2.01 & 6.89 & $3.3 Y$ & $4.6 /$ & 1 & 11.44 & 11.73 & 12.02 & 12.43 & 12.88 & 13.26 & 13.54 & 13.78 & 14.00 \\
\hline 1 & $\mathrm{H}$ & CC & 27 & 27.1 & & & 53.61 & 1.96 & 7.18 & $3.5 Y$ & 5.21 & 1 & 15.24 & 15.62 & 16.03 & 16.55 & 17.11 & 17.63 & 18.00 & 18.31 & 18.62 \\
\hline 1 & $\mathrm{H}$ & CC & 32 & 32.1 & & & 52.44 & 2.27 & 8.05 & $3.4 \mathrm{Y}$ & $5.0 /$ & 1.1 & 13.70 & 14.10 & 14.55 & 15.12 & 15.72 & 16.25 & 16.66 & 17.00 & 17.32 \\
\hline 1 & $\mathrm{H}$ & CC & 37 & 37.1 & & & 52.84 & 2.10 & 7.07 & $3.2 \mathrm{Y}$ & $5.1 /$ & 1 & 14.71 & 15.09 & 15.50 & 16.03 & 16.59 & 17.06 & 17.43 & 17.74 & 18.03 \\
\hline 1 & $\mathrm{H}$ & CC & 42 & 42.1 & & & 48.84 & 2.75 & 9.90 & $3.5 Y$ & $4.7 /$ & 1.4 & 10.13 & 10.60 & 11.06 & 11.64 & 12.28 & 12.84 & 13.27 & 13.62 & 13.95 \\
\hline 1 & $\mathrm{H}$ & CC & 47 & 47.1 & & & 55.01 & 2.51 & 9.66 & $3.6 \mathrm{Y}$ & $5.3 /$ & 1.3 & 14.19 & 14.71 & 15.25 & 15.96 & 16.75 & 17.45 & 18.00 & 18.45 & 18.86 \\
\hline 1 & $\mathrm{H}$ & CC & 52 & 52.1 & & & 53.35 & 1.52 & 7.43 & $4.5 \mathrm{Y}$ & $5.1 /$ & 1 & 14.24 & 14.76 & 15.30 & 15.99 & 16.70 & 17.27 & 17.70 & 18.07 & 18.41 \\
\hline 1 & $\mathrm{H}$ & CC & 57 & 57.1 & & & 52.63 & 1.91 & 8.02 & $4.0 \mathrm{Y}$ & $5.1 /$ & 1.1 & 13.54 & 14.02 & 14.51 & 15.14 & 15.83 & 16.43 & 16.85 & 17.20 & 17.54 \\
\hline 1 & $\mathrm{H}$ & CC & 62 & 62.1 & & & 51.58 & 2.68 & 10.66 & $3.8 \mathrm{Y}$ & $4.9 /$ & 1.5 & 10.89 & 11.48 & 12.10 & 12.86 & 13.68 & 14.38 & 14.90 & 15.34 & 15.75 \\
\hline 1 & $\mathrm{H}$ & CC & 67 & 67.1 & & & 49.30 & 1.67 & 7.36 & $4.3 Y$ & $4.7 /$ & 1 & 11.72 & 12.15 & 12.59 & 13.15 & 13.75 & 14.27 & 14.65 & 14.95 & 15.22 \\
\hline
\end{tabular}

\begin{tabular}{|c|c|c|c|c|c|c|c|c|c|c|c|c|c|c|c|c|c|c|c|c|c|}
\hline 490 & 500 & 510 & 520 & 530 & 540 & 550 & 560 & 570 & 580 & 590 & 600 & 610 & 620 & 630 & 640 & 650 & 660 & 670 & 680 & 690 & 700 \\
\hline 10.49 & 10.74 & 11.02 & 11.29 & 11.58 & 11.89 & 12.22 & 12.50 & 12.79 & 13.02 & 13.25 & 13.46 & 13.65 & 13.82 & 14.01 & 14.20 & 14.40 & 14.55 & 14.67 & 14.78 & 14.84 & 14.91 \\
\hline 13.08 & 13.35 & 13.67 & 13.99 & 14.30 & 14.62 & 14.92 & 15.18 & 15.45 & 15.64 & 15.79 & 15.91 & 16.04 & 16.12 & 16.25 & 16.31 & 16.41 & 16.54 & 16.59 & 16.63 & 16.70 & 16.75 \\
\hline 15.10 & 15.36 & 15.66 & 15.97 & 16.28 & 16.54 & 16.80 & 17.03 & 17.25 & 17.37 & 17.44 & 17.49 & 17.57 & 17.58 & 17.62 & 17.62 & 17.64 & 17.69 & 17.73 & 17.73 & 17.75 & 17.80 \\
\hline 15.55 & 15.76 & 16.04 & 16.29 & 16.56 & 16.79 & 17.03 & 17.22 & 17.42 & 17.52 & 17.63 & 17.68 & 17.73 & 17.77 & 17.82 & 17.83 & 17.88 & 17.94 & 17.93 & 17.93 & 17.95 & 18.00 \\
\hline 14.26 & 14.55 & 14.87 & 15.18 & 15.51 & 15.82 & 16.12 & 16.39 & 16.61 & 16.82 & 16.98 & 17.08 & 17.15 & 17.19 & 17.25 & 17.26 & 17.33 & 17.42 & 17.47 & 17.49 & 17.52 & 17.58 \\
\hline 18.96 & 19.32 & 19.70 & 20.10 & 20.53 & 20.90 & 21.26 & 21.60 & 21.91 & 22.10 & 22.28 & 22.38 & 22.50 & 22.51 & 22.59 & 22.63 & 22.68 & 22.73 & 22.79 & 22.84 & 22.88 & 22.90 \\
\hline 17.68 & 18.07 & 18.49 & 18.87 & 19.35 & 19.74 & 20.14 & 20.50 & 20.83 & 21.08 & 21.27 & 21.39 & 21.50 & 21.57 & 21.64 & 21.67 & 21.76 & 21.83 & 21.88 & 21.91 & 21.94 & 22.02 \\
\hline 18.34 & 18.68 & 19.04 & 19.39 & 19.79 & 20.16 & 20.50 & 20.84 & 21.15 & 21.41 & 21.60 & 21.70 & 21.81 & 21.87 & 21.94 & 21.93 & 22.00 & 22.06 & 22.09 & 22.13 & 22.18 & 22.22 \\
\hline 14.33 & 14.75 & 15.24 & 15.71 & 16.18 & 16.61 & 17.01 & 17.44 & 17.79 & 18.04 & 18.26 & 18.38 & 18.50 & 18.58 & 18.67 & 18.71 & 18.81 & 18.89 & 18.93 & 18.97 & 19.01 & 19.04 \\
\hline 19.29 & 19.81 & 20.38 & 20.88 & 21.47 & 21.99 & 22.47 & 22.92 & 23.33 & 23.66 & 23.88 & 24.00 & 24.13 & 24.20 & 24.28 & 24.24 & 24.33 & 24.45 & 24.50 & 24.50 & 24.53 & 24.62 \\
\hline 18.76 & 19.14 & 19.57 & 19.97 & 20.42 & 20.80 & 21.14 & 21.44 & 21.72 & 21.91 & 22.01 & 22.05 & 22.09 & 22.12 & 22.15 & 22.11 & 22.14 & 22.18 & 22.20 & 22.23 & 22.25 & 22.28 \\
\hline 17.92 & 18.31 & 18.75 & 19.15 & 19.59 & 20.00 & 20.38 & 20.75 & 21.06 & 21.27 & 21.45 & 21.51 & 21.57 & 21.60 & 21.64 & 21.63 & 21.66 & 21.70 & 21.74 & 21.80 & 21.83 & 21.84 \\
\hline 16.20 & 16.70 & 17.25 & 17.76 & 18.34 & 18.86 & 19.32 & 19.76 & 20.19 & 20.47 & 20.69 & 20.81 & 20.92 & 20.97 & 21.04 & 21.07 & 21.15 & 21.24 & 21.29 & 21.35 & 21.40 & 21.46 \\
\hline 15.52 & 15.86 & 16.23 & 16.57 & 16.95 & 17.28 & 17.61 & 17.87 & 18.13 & 18.29 & 18.42 & 18.47 & 18.53 & 18.55 & 18.59 & 18.58 & 18.62 & 18.64 & 18.68 & 18.72 & 18.74 & 18.77 \\
\hline
\end{tabular}

Note: Only a portion of this table appears here. The complete table is available in ASCII format.

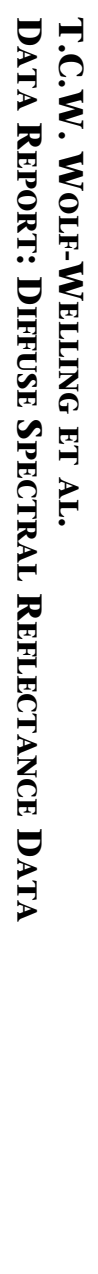


Table T6. Spectral reflectance data, Hole 1096A.

\begin{tabular}{|c|c|c|c|c|c|c|c|c|c|c|c|c|c|c|c|c|c|c|c|c|c|}
\hline Core & $\begin{array}{l}\text { Core } \\
\text { type }\end{array}$ & Section & $\begin{array}{l}\text { Interval } \\
\text { top } \\
(\mathrm{cm})\end{array}$ & $\begin{array}{l}\text { Interval } \\
\text { bottom } \\
(\mathrm{cm})\end{array}$ & $\begin{array}{l}\text { Curator } \\
\text { depth } \\
\text { (mbsf) }\end{array}$ & $\begin{array}{l}\text { Recovery- } \\
\text { corrected } \\
\text { depth } \\
\text { (mbsf) }\end{array}$ & $L^{*}$ & $a^{*}$ & $b^{*}$ & Munsell & Munsell & Munsell & 400 & 410 & 420 & 430 & 440 & 450 & 460 & 470 & 480 \\
\hline 1 & $\mathrm{H}$ & 1 & 2 & 2.1 & 0.02 & 0.02 & 44.20 & 2.65 & 5.79 & $0.7 Y$ & $4.2 /$ & 0.8 & 10.66 & 10.77 & 10.94 & 11.17 & 11.37 & 11.58 & 11.75 & 11.90 & 12.05 \\
\hline 1 & $\mathrm{H}$ & 1 & 7 & 7.1 & 0.07 & 0.07 & 41.87 & 2.00 & 4.60 & $1.1 \mathrm{Y}$ & $4.0 /$ & 0.6 & 10.07 & 10.10 & 10.17 & 10.31 & 10.48 & 10.65 & 10.76 & 10.87 & 11.00 \\
\hline 1 & $\mathrm{H}$ & 1 & 12 & 12.1 & 0.12 & 0.12 & 45.04 & 3.54 & 9.94 & $2.3 \mathrm{Y}$ & $4.3 /$ & 1.4 & 8.59 & 8.87 & 9.21 & 9.64 & 10.08 & 10.48 & 10.82 & 11.11 & 11.39 \\
\hline 1 & $\mathrm{H}$ & 1 & 17 & 17.1 & 0.17 & 0.17 & 45.50 & 0.95 & 4.36 & $4.5 \mathrm{Y}$ & $4.4 /$ & 0.6 & 11.68 & 11.86 & 12.11 & 12.40 & 12.71 & 12.97 & 13.17 & 13.33 & 13.48 \\
\hline 1 & $\mathrm{H}$ & 1 & 22 & 22.1 & 0.22 & 0.22 & 39.27 & 0.54 & 4.70 & $7.2 \mathrm{Y}$ & $3.8 /$ & 0.7 & 7.54 & 7.82 & 8.17 & 8.55 & 8.89 & 9.16 & 9.34 & 9.48 & 9.63 \\
\hline 1 & $\mathrm{H}$ & 1 & 27 & 27.1 & 0.27 & 0.27 & 43.96 & 0.68 & 5.62 & $6.9 \mathrm{Y}$ & $4.2 /$ & 0.8 & 9.38 & 9.74 & 10.15 & 10.65 & 11.11 & 11.46 & 11.72 & 11.95 & 12.19 \\
\hline 1 & $\mathrm{H}$ & 1 & 32 & 32.1 & 0.32 & 0.32 & 48.68 & 0.43 & 3.70 & $7.0 \mathrm{Y}$ & $4.7 /$ & 0.5 & 13.85 & 14.15 & 14.46 & 14.83 & 15.20 & 15.50 & 15.71 & 15.89 & 16.06 \\
\hline 1 & $\mathrm{H}$ & 1 & 37 & 37.1 & 0.37 & 0.37 & 49.19 & 0.76 & 4.84 & $5.7 Y$ & $4.7 /$ & 0.7 & 13.32 & 13.68 & 14.06 & 14.52 & 14.97 & 15.33 & 15.60 & 15.84 & 16.07 \\
\hline 1 & $\mathrm{H}$ & 1 & 42 & 42.1 & 0.42 & 0.42 & 48.70 & 0.30 & 4.62 & $8.2 \mathrm{Y}$ & $4.7 /$ & 0.7 & 12.80 & 13.21 & 13.65 & 14.15 & 14.65 & 15.07 & 15.36 & 15.60 & 15.82 \\
\hline 1 & $\mathrm{H}$ & 1 & 47 & 47.1 & 0.47 & 0.47 & 46.95 & 0.15 & 3.41 & $8.4 \mathrm{Y}$ & $4.5 /$ & 0.5 & 12.65 & 13.00 & 13.36 & 13.74 & 14.08 & 14.37 & 14.58 & 14.75 & 14.90 \\
\hline 1 & $\mathrm{H}$ & 1 & 52 & 52.1 & 0.52 & 0.52 & 47.14 & 0.69 & 5.34 & $6.7 \mathrm{Y}$ & $4.5 /$ & 0.8 & 11.65 & 11.98 & 12.31 & 12.75 & 13.21 & 13.61 & 13.91 & 14.19 & 14.44 \\
\hline 1 & $\mathrm{H}$ & 1 & 57 & 57.1 & 0.57 & 0.57 & 46.93 & -0.05 & 4.08 & $9.6 \mathrm{Y}$ & $4.5 /$ & 0.6 & 11.93 & 12.31 & 12.70 & 13.19 & 13.65 & 14.01 & 14.30 & 14.54 & 14.75 \\
\hline 1 & $\mathrm{H}$ & 1 & 62 & 62.1 & 0.62 & 0.62 & 45.87 & -0.23 & 3.40 & $0.4 \mathrm{GY}$ & $4.4 /$ & 0.5 & 11.61 & 11.96 & 12.36 & 12.84 & 13.27 & 13.60 & 13.85 & 14.06 & 14.23 \\
\hline 1 & $\mathrm{H}$ & 1 & 67 & 67.1 & 0.67 & 0.67 & 47.62 & 0.62 & 4.91 & $6.8 \mathrm{Y}$ & $4.6 /$ & 0.7 & 12.02 & 12.43 & 12.84 & 13.30 & 13.76 & 14.15 & 14.46 & 14.70 & 14.90 \\
\hline
\end{tabular}

\begin{tabular}{|c|c|c|c|c|c|c|c|c|c|c|c|c|c|c|c|c|c|c|c|c|c|}
\hline 490 & 500 & 510 & 520 & 530 & 540 & 550 & 560 & 570 & 580 & 590 & 600 & 610 & 620 & 630 & 640 & 650 & 660 & 670 & 680 & 690 & 700 \\
\hline 12.23 & 12.42 & 12.65 & 12.87 & 13.11 & 13.33 & 13.59 & 13.83 & 14.03 & 14.21 & 14.40 & 14.54 & 14.69 & 14.84 & 15.03 & 15.14 & 15.29 & 15.43 & 15.56 & 15.65 & 15.72 & 15.76 \\
\hline 11.15 & 11.27 & 11.44 & 11.63 & 11.80 & 11.98 & 12.15 & 12.31 & 12.47 & 12.61 & 12.74 & 12.84 & 12.93 & 13.01 & 13.13 & 13.24 & 13.32 & 13.40 & 13.49 & 13.57 & 13.59 & 13.63 \\
\hline 11.70 & 12.04 & 12.44 & 12.84 & 13.27 & 13.66 & 14.05 & 14.42 & 14.78 & 15.05 & 15.30 & 15.46 & 15.63 & 15.81 & 15.99 & 16.11 & 16.27 & 16.42 & 16.54 & 16.64 & 16.70 & 16.77 \\
\hline 13.66 & 13.85 & 14.05 & 14.24 & 14.44 & 14.60 & 14.80 & 14.93 & 15.06 & 15.15 & 15.21 & 15.22 & 15.26 & 15.26 & 15.28 & 15.27 & 15.28 & 15.30 & 15.32 & 15.32 & 15.32 & 15.33 \\
\hline 9.80 & 9.98 & 10.18 & 10.35 & 10.50 & 10.66 & 10.81 & 10.90 & 10.98 & 11.02 & 11.05 & 11.02 & 11.01 & 11.01 & 11.01 & 10.99 & 10.97 & 10.96 & 10.95 & 10.94 & 10.93 & 10.91 \\
\hline 12.41 & 12.65 & 12.91 & 13.15 & 13.38 & 13.58 & 13.79 & 13.90 & 14.01 & 14.12 & 14.15 & 14.12 & 14.10 & 14.12 & 14.10 & 14.04 & 14.04 & 14.00 & 13.98 & 13.96 & 13.90 & 13.86 \\
\hline 16.27 & 16.43 & 16.62 & 16.82 & 17.03 & 17.17 & 17.33 & 17.42 & 17.51 & 17.54 & 17.58 & 17.57 & 17.54 & 17.56 & 17.57 & 17.53 & 17.49 & 17.49 & 17.51 & 17.47 & 17.41 & 17.40 \\
\hline 16.31 & 16.51 & 16.76 & 17.01 & 17.27 & 17.49 & 17.67 & 17.83 & 17.97 & 18.05 & 18.09 & 18.11 & 18.11 & 18.08 & 18.11 & 18.09 & 18.11 & 18.07 & 18.05 & 18.05 & 18.03 & 18.00 \\
\hline 16.07 & 16.32 & 16.57 & 16.77 & 17.01 & 17.20 & 17.38 & 17.49 & 17.57 & 17.63 & 17.65 & 17.61 & 17.59 & 17.52 & 17.54 & 17.49 & 17.42 & 17.36 & 17.35 & 17.31 & 17.24 & 17.23 \\
\hline 15.07 & 15.23 & 15.39 & 15.56 & 15.76 & 15.90 & 16.01 & 16.09 & 16.17 & 16.19 & 16.19 & 16.15 & 16.12 & 16.10 & 16.06 & 15.98 & 15.97 & 15.95 & 15.92 & 15.87 & 15.83 & 15.82 \\
\hline 14.68 & 14.90 & 15.16 & 15.42 & 15.66 & 15.87 & 16.09 & 16.24 & 16.34 & 16.42 & 16.46 & 16.44 & 16.45 & 16.45 & 16.46 & 16.42 & 16.41 & 16.42 & 16.41 & 16.36 & 16.32 & 16.31 \\
\hline 14.98 & 15.17 & 15.39 & 15.56 & 15.77 & 15.92 & 16.03 & 16.12 & 16.18 & 16.20 & 16.19 & 16.11 & 16.04 & 16.02 & 16.00 & 15.94 & 15.88 & 15.84 & 15.81 & 15.79 & 15.73 & 15.66 \\
\hline 14.40 & 14.57 & 14.75 & 14.89 & 15.05 & 15.19 & 15.27 & 15.32 & 15.38 & 15.38 & 15.34 & 15.25 & 15.23 & 15.18 & 15.12 & 15.03 & 14.99 & 14.94 & 14.91 & 14.88 & 14.81 & 14.77 \\
\hline 15.13 & 15.36 & 15.60 & 15.83 & 16.06 & 16.26 & 16.42 & 16.59 & 16.74 & 16.80 & 16.81 & 16.83 & 16.81 & 16.79 & 16.78 & 16.72 & 16.70 & 16.69 & 16.70 & 16.68 & 16.66 & 16.68 \\
\hline
\end{tabular}

Note: Only a portion of this table appears here. The complete table is available in ASCII format. 
Table T7. Spectral reflectance data, Hole 1096B.

\begin{tabular}{|c|c|c|c|c|c|c|c|c|c|c|c|c|c|c|c|c|c|c|c|c|c|}
\hline Core & $\begin{array}{l}\text { Core } \\
\text { type }\end{array}$ & Section & $\begin{array}{l}\text { Interval } \\
\text { top } \\
(\mathrm{cm})\end{array}$ & $\begin{array}{l}\text { Interval } \\
\text { bottom } \\
(\mathrm{cm})\end{array}$ & $\begin{array}{c}\text { Curator } \\
\text { depth } \\
\text { (mbsf) }\end{array}$ & $\begin{array}{l}\text { Recovery- } \\
\text { corrected } \\
\text { depth } \\
\text { (mbsf) }\end{array}$ & $L^{*}$ & $a^{*}$ & $b^{*}$ & Munsell & Munsell & Munsell & 400 & 410 & 420 & 430 & 440 & 450 & 460 & 470 & 480 \\
\hline 1 & $\mathrm{H}$ & 1 & 2 & 2.1 & 0.02 & 0.02 & 30.80 & 3.73 & 8.60 & $1.6 \mathrm{Y}$ & 2.9/ & 1.3 & 3.75 & 3.85 & 3.98 & 4.17 & 4.37 & 4.52 & 4.66 & 4.79 & 4.93 \\
\hline 1 & $\mathrm{H}$ & 1 & 7 & 7.1 & 0.07 & 0.07 & 36.08 & 3.99 & 9.60 & $1.6 \mathrm{Y}$ & $3.4 /$ & 1.4 & 5.21 & 5.34 & 5.47 & 5.70 & 6.00 & 6.24 & 6.42 & 6.61 & 6.79 \\
\hline 1 & $\mathrm{H}$ & 1 & 12 & 12.1 & 0.12 & 0.12 & 40.15 & 3.93 & 10.98 & $2.3 \mathrm{Y}$ & $3.8 /$ & 1.5 & 5.96 & 6.20 & 6.49 & 6.85 & 7.24 & 7.59 & 7.89 & 8.16 & 8.41 \\
\hline 1 & $\mathrm{H}$ & 1 & 17 & 17.1 & 0.17 & 0.17 & 43.16 & 2.65 & 8.92 & $3.4 \mathrm{Y}$ & $4.1 /$ & 1.2 & 7.85 & 8.16 & 8.51 & 8.95 & 9.38 & 9.78 & 10.10 & 10.37 & 10.62 \\
\hline 1 & $\mathrm{H}$ & 1 & 22 & 22.1 & 0.22 & 0.22 & 44.79 & 2.19 & 7.78 & $3.6 \mathrm{Y}$ & $4.3 /$ & 1.1 & 9.12 & 9.43 & 9.80 & 10.29 & 10.76 & 11.15 & 11.46 & 11.72 & 11.94 \\
\hline 1 & $\mathrm{H}$ & 1 & 27 & 27.1 & 0.27 & 0.27 & 46.48 & 2.27 & 8.26 & $3.6 \mathrm{Y}$ & $4.5 /$ & 1.1 & 9.66 & 10.05 & 10.47 & 11.01 & 11.57 & 12.02 & 12.33 & 12.63 & 12.91 \\
\hline 1 & $\mathrm{H}$ & 1 & 32 & 32.1 & 0.32 & 0.32 & 48.04 & 0.93 & 6.40 & $6.1 \mathrm{Y}$ & $4.6 /$ & 0.9 & 11.39 & 11.83 & 12.24 & 12.78 & 13.34 & 13.80 & 14.13 & 14.40 & 14.68 \\
\hline 1 & $\mathrm{H}$ & 1 & 37 & 37.1 & 0.37 & 0.37 & 48.45 & 0.47 & 4.53 & $7.3 \mathrm{Y}$ & $4.7 /$ & 0.7 & 12.98 & 13.30 & 13.66 & 14.12 & 14.56 & 14.93 & 15.20 & 15.43 & 15.62 \\
\hline 1 & $\mathrm{H}$ & 1 & 42 & 42.1 & 0.42 & 0.42 & 46.68 & -0.01 & 4.13 & $9.3 Y$ & $4.5 /$ & 0.6 & 11.87 & 12.22 & 12.59 & 13.03 & 13.49 & 13.86 & 14.12 & 14.33 & 14.51 \\
\hline 1 & $\mathrm{H}$ & 1 & 47 & 47.1 & 0.47 & 0.47 & 47.02 & 0.60 & 6.74 & $7.7 Y$ & $4.5 /$ & 1 & 10.14 & 10.68 & 11.19 & 11.80 & 12.44 & 12.95 & 13.32 & 13.62 & 13.92 \\
\hline 1 & $\mathrm{H}$ & 1 & 52 & 52.1 & 0.52 & 0.52 & 48.63 & 0.25 & 5.75 & $8.5 \mathrm{Y}$ & $4.7 /$ & 0.9 & 11.74 & 12.24 & 12.75 & 13.34 & 13.96 & 14.48 & 14.87 & 15.17 & 15.44 \\
\hline 1 & $\mathrm{H}$ & 1 & 57 & 57.1 & 0.57 & 0.57 & 44.29 & 0.51 & 5.41 & $7.6 \mathrm{Y}$ & $4.3 /$ & 0.8 & 9.60 & 9.98 & 10.40 & 10.89 & 11.36 & 11.74 & 12.03 & 12.26 & 12.48 \\
\hline 1 & $\mathrm{H}$ & 1 & 62 & 62.1 & 0.62 & 0.62 & 47.13 & 0.19 & 4.59 & $8.6 \mathrm{Y}$ & $4.6 /$ & 0.7 & 11.75 & 12.16 & 12.57 & 13.06 & 13.54 & 13.94 & 14.26 & 14.51 & 14.70 \\
\hline 1 & $\mathrm{H}$ & 1 & 67 & 67.1 & 0.67 & 0.67 & 46.34 & -0.18 & 3.66 & $0.1 \mathrm{GY}$ & $4.5 /$ & 0.6 & 11.77 & 12.14 & 12.53 & 13.01 & 13.46 & 13.81 & 14.08 & 14.30 & 14.47 \\
\hline
\end{tabular}

\begin{tabular}{|c|c|c|c|c|c|c|c|c|c|c|c|c|c|c|c|c|c|c|c|c|c|}
\hline 490 & 500 & 510 & 520 & 530 & 540 & 550 & 560 & 570 & 580 & 590 & 600 & 610 & 620 & 630 & 640 & 650 & 660 & 670 & 680 & 690 & 700 \\
\hline 5.09 & 5.26 & 5.42 & 5.60 & 5.82 & 6.02 & 6.23 & 6.42 & 6.62 & 6.79 & 6.94 & 7.06 & 7.19 & 7.31 & 7.45 & 7.57 & 7.68 & 7.79 & 7.90 & 8.00 & 8.04 & 8.07 \\
\hline 7.00 & 7.22 & 7.48 & 7.75 & 8.04 & 8.33 & 8.62 & 8.89 & 9.12 & 9.36 & 9.58 & 9.70 & 9.87 & 10.06 & 10.22 & 10.35 & 10.52 & 10.68 & 10.80 & 10.89 & 10.93 & 10.97 \\
\hline 8.69 & 9.00 & 9.35 & 9.72 & 10.13 & 10.49 & 10.86 & 11.22 & 11.51 & 11.75 & 12.00 & 12.16 & 12.35 & 12.51 & 12.65 & 12.79 & 12.95 & 13.10 & 13.24 & 13.34 & 13.38 & 13.46 \\
\hline 10.89 & 11.22 & 11.57 & 11.90 & 12.27 & 12.59 & 12.93 & 13.23 & 13.49 & 13.69 & 13.83 & 13.93 & 14.06 & 14.15 & 14.24 & 14.31 & 14.43 & 14.53 & 14.60 & 14.66 & 14.71 & 14.75 \\
\hline 12.21 & 12.52 & 12.83 & 13.13 & 13.45 & 13.79 & 14.10 & 14.37 & 14.63 & 14.82 & 14.94 & 15.02 & 15.11 & 15.16 & 15.24 & 15.27 & 15.34 & 15.38 & 15.46 & 15.51 & 15.51 & 15.60 \\
\hline 13.19 & 13.52 & 13.90 & 14.26 & 14.62 & 14.96 & 15.30 & 15.60 & 15.87 & 16.10 & 16.23 & 16.34 & 16.44 & 16.49 & 16.56 & 16.58 & 16.63 & 16.70 & 16.77 & 16.81 & 16.82 & 16.89 \\
\hline 14.98 & 15.27 & 15.60 & 15.92 & 16.23 & 16.52 & 16.78 & 16.95 & 17.07 & 17.19 & 17.25 & 17.24 & 17.26 & 17.27 & 17.26 & 17.23 & 17.23 & 17.23 & 17.22 & 17.21 & 17.18 & 17.17 \\
\hline 15.85 & 16.08 & 16.31 & 16.52 & 16.75 & 16.97 & 17.15 & 17.27 & 17.37 & 17.43 & 17.44 & 17.43 & 17.42 & 17.39 & 17.38 & 17.33 & 17.32 & 17.30 & 17.27 & 17.23 & 17.16 & 17.11 \\
\hline 14.71 & 14.90 & 15.14 & 15.36 & 15.55 & 15.72 & 15.85 & 15.96 & 16.01 & 16.03 & 16.01 & 15.93 & 15.91 & 15.85 & 15.84 & 15.73 & 15.66 & 15.63 & 15.57 & 15.52 & 15.45 & 15.37 \\
\hline 14.24 & 14.57 & 14.91 & 15.19 & 15.52 & 15.81 & 16.07 & 16.21 & 16.34 & 16.40 & 16.46 & 16.41 & 16.39 & 16.34 & 16.32 & 16.27 & 16.23 & 16.21 & 16.18 & 16.12 & 16.06 & 16.03 \\
\hline 15.72 & 16.01 & 16.33 & 16.62 & 16.92 & 17.15 & 17.34 & 17.51 & 17.62 & 17.64 & 17.62 & 17.57 & 17.56 & 17.49 & 17.44 & 17.38 & 17.35 & 17.30 & 17.28 & 17.24 & 17.19 & 17.16 \\
\hline 12.72 & 12.95 & 13.19 & 13.41 & 13.66 & 13.86 & 14.05 & 14.15 & 14.29 & 14.32 & 14.32 & 14.31 & 14.29 & 14.28 & 14.29 & 14.23 & 14.21 & 14.17 & 14.13 & 14.13 & 14.07 & 14.02 \\
\hline 14.92 & 15.15 & 15.37 & 15.56 & 15.83 & 16.03 & 16.16 & 16.27 & 16.39 & 16.37 & 16.37 & 16.33 & 16.32 & 16.27 & 16.23 & 16.18 & 16.13 & 16.11 & 16.09 & 16.03 & 15.97 & 15.96 \\
\hline 14.67 & 14.84 & 15.02 & 15.20 & 15.37 & 15.52 & 15.61 & 15.68 & 15.72 & 15.73 & 15.72 & 15.65 & 15.59 & 15.51 & 15.49 & 15.42 & 15.36 & 15.32 & 15.29 & 15.28 & 15.23 & 15.16 \\
\hline
\end{tabular}

Note: Only a portion of this table appears here. The complete table is available in ASCII format. 
Table T8. Spectral reflectance data, Hole 1096C.

\begin{tabular}{|c|c|c|c|c|c|c|c|c|c|c|c|c|c|c|c|c|c|c|c|c|c|}
\hline Core & $\begin{array}{l}\text { Core } \\
\text { type }\end{array}$ & Section & $\begin{array}{l}\text { Interval } \\
\text { top } \\
(\mathrm{cm})\end{array}$ & $\begin{array}{l}\text { Interval } \\
\text { bottom } \\
(\mathrm{cm})\end{array}$ & $\begin{array}{l}\text { Curator } \\
\text { depth } \\
\text { (mbsf) }\end{array}$ & $\begin{array}{l}\text { Recovery- } \\
\text { corrected } \\
\text { depth } \\
\text { (mbsf) }\end{array}$ & $\mathrm{L}^{*}$ & $a^{*}$ & $b^{*}$ & Munsell & Munsell & Munsell & 400 & 410 & 420 & 430 & 440 & 450 & 460 & 470 & 480 \\
\hline 1 & $\mathrm{H}$ & 1 & 2 & 2.1 & 114.02 & 114.02 & 41.54 & -0.17 & 1.14 & $10.0 \mathrm{Y}$ & $4.0 /$ & 0.2 & 11.42 & 11.45 & 11.51 & 11.61 & 11.68 & 11.72 & 11.76 & 11.84 & 11.91 \\
\hline 1 & $\mathrm{H}$ & 1 & 7 & 7.1 & 114.07 & 114.07 & 42.13 & -0.08 & 1.43 & $9.2 \mathrm{Y}$ & $4.1 /$ & 0.2 & 11.58 & 11.61 & 11.72 & 11.84 & 11.90 & 11.95 & 12.02 & 12.12 & 12.20 \\
\hline 1 & $\mathrm{H}$ & 1 & 12 & 12.1 & 114.12 & 114.12 & 42.79 & -1.88 & 0.48 & $3.4 \mathrm{G}$ & 4.21 & 0.4 & 12.06 & 12.14 & 12.29 & 12.46 & 12.59 & 12.69 & 12.80 & 12.94 & 13.10 \\
\hline 1 & $\mathrm{H}$ & 1 & 17 & 17.1 & 114.17 & 114.17 & 43.56 & -2.00 & 0.21 & $5.8 \mathrm{G}$ & 4.21 & 0.4 & 12.74 & 12.80 & 12.93 & 13.11 & 13.23 & 13.30 & 13.42 & 13.57 & 13.71 \\
\hline 1 & $\mathrm{H}$ & 1 & 22 & 22.1 & 114.22 & 114.21 & 45.16 & -1.24 & -0.40 & $4.6 \mathrm{BC}$ & $4.4 /$ & 0.2 & 14.53 & 14.54 & 14.60 & 14.69 & 14.75 & 14.78 & 14.82 & 14.88 & 14.93 \\
\hline 1 & $\mathrm{H}$ & 1 & 27 & 27.1 & 114.27 & 114.26 & 25.17 & -0.50 & 0.16 & $2.5 \mathrm{G}$ & $2.5 /$ & 0.2 & 4.33 & 4.36 & 4.36 & 4.39 & 4.42 & 4.43 & 4.44 & 4.45 & 4.46 \\
\hline 1 & $\mathrm{H}$ & 1 & 32 & 32.1 & 114.32 & 114.31 & 26.52 & -0.13 & 0.22 & $5.3 \mathrm{GY}$ & $2.6 /$ & 0.1 & 4.91 & 4.88 & 4.87 & 4.87 & 4.89 & 4.88 & 4.86 & 4.88 & 4.88 \\
\hline 1 & $\mathrm{H}$ & 1 & 37 & 37.1 & 114.37 & 114.36 & 56.51 & 14.74 & 6.96 & $1.8 \mathrm{R}$ & $5.3 /$ & 3.1 & 21.06 & 21.17 & 21.31 & 21.34 & 21.22 & 21.04 & 20.70 & 20.30 & 19.89 \\
\hline 1 & $\mathrm{H}$ & 1 & 42 & 42.1 & 114.42 & 114.41 & 36.70 & -0.93 & 1.15 & $7.0 \mathrm{GY}$ & $3.6 /$ & 0.3 & 8.43 & 8.51 & 8.61 & 8.76 & 8.85 & 8.92 & 9.00 & 9.10 & 9.20 \\
\hline 1 & $\mathrm{H}$ & 1 & 47 & 47.1 & 114.47 & 114.46 & 50.56 & -2.32 & 0.58 & $3.7 \mathrm{G}$ & $4.9 /$ & 0.5 & 17.43 & 17.55 & 17.74 & 18.00 & 18.20 & 18.37 & 18.55 & 18.79 & 19.02 \\
\hline 1 & $\mathrm{H}$ & 1 & 52 & 52.1 & 114.52 & 114.51 & 41.67 & -0.50 & 0.80 & $5.7 \mathrm{GY}$ & $4.0 /$ & 0.2 & 11.66 & 11.71 & 11.73 & 11.80 & 11.87 & 11.91 & 11.96 & 12.04 & 12.13 \\
\hline 1 & $\mathrm{H}$ & 1 & 57 & 57.1 & 114.57 & 114.55 & 41.87 & -0.08 & 1.73 & $8.2 \mathrm{Y}$ & $4.1 /$ & 0.3 & 11.30 & 11.33 & 11.41 & 11.52 & 11.61 & 11.67 & 11.74 & 11.86 & 11.97 \\
\hline 1 & $\mathrm{H}$ & 1 & 62 & 62.1 & 114.62 & 114.60 & 45.88 & -0.19 & 1.28 & $0.2 \mathrm{GY}$ & $4.4 /$ & 0.2 & 14.20 & 14.24 & 14.32 & 14.42 & 14.48 & 14.55 & 14.61 & 14.69 & 14.79 \\
\hline 1 & $\mathrm{H}$ & 1 & 67 & 67.1 & 114.67 & 114.65 & 42.41 & -0.21 & 1.33 & $0.2 \mathrm{GY}$ & $4.1 /$ & 0.2 & 11.80 & 11.82 & 11.92 & 12.05 & 12.13 & 12.18 & 12.24 & 12.33 & 12.41 \\
\hline
\end{tabular}

\begin{tabular}{|c|c|c|c|c|c|c|c|c|c|c|c|c|c|c|c|c|c|c|c|c|c|}
\hline 490 & 500 & 510 & 520 & 530 & 540 & 550 & 560 & 570 & 580 & 590 & 600 & 610 & 620 & 630 & 640 & 650 & 660 & 670 & 680 & 690 & 700 \\
\hline 11.97 & 12.00 & 12.07 & 12.11 & 12.10 & 12.14 & 12.22 & 12.25 & 12.32 & 12.36 & 12.37 & 12.30 & 12.23 & 12.18 & 12.15 & 12.02 & 11.88 & 11.80 & 11.74 & 11.66 & 11.55 & 11.47 \\
\hline 12.28 & 12.33 & 12.40 & 12.46 & 12.48 & 12.48 & 12.57 & 12.65 & 12.71 & 12.73 & 12.73 & 12.69 & 12.67 & 12.58 & 12.53 & 12.45 & 12.35 & 12.25 & 12.20 & 12.13 & 12.04 & 11.97 \\
\hline 13.23 & 13.29 & 13.36 & 13.42 & 13.40 & 13.36 & 13.34 & 13.26 & 13.16 & 13.07 & 12.95 & 12.81 & 12.64 & 12.52 & 12.41 & 12.23 & 12.08 & 11.95 & 11.80 & 11.64 & 11.49 & 11.36 \\
\hline 13.84 & 13.90 & 13.98 & 13.99 & 13.95 & 13.92 & 13.87 & 13.79 & 13.71 & 13.56 & 13.45 & 13.29 & 13.14 & 12.98 & 12.86 & 12.69 & 12.49 & 12.33 & 12.21 & 12.08 & 11.92 & 11.79 \\
\hline 14.98 & 15.00 & 15.01 & 15.00 & 14.96 & 14.92 & 14.88 & 14.80 & 14.75 & 14.65 & 14.57 & 14.47 & 14.37 & 14.28 & 14.22 & 14.11 & 13.99 & 13.90 & 13.83 & 13.75 & 13.64 & 13.54 \\
\hline 4.50 & 4.50 & 4.50 & 4.54 & 4.52 & 4.50 & 4.50 & 4.52 & 4.49 & 4.49 & 4.47 & 4.44 & 4.42 & 4.38 & 4.40 & 4.38 & 4.34 & 4.32 & 4.33 & 4.30 & 4.27 & 4.27 \\
\hline 4.90 & 4.88 & 4.89 & 4.95 & 4.94 & 4.94 & 4.95 & 4.96 & 4.94 & 4.91 & 4.93 & 4.91 & 4.92 & 4.91 & 4.93 & 4.89 & 4.87 & 4.87 & 4.88 & 4.87 & 4.84 & 4.86 \\
\hline 19.55 & 19.35 & 19.31 & 19.31 & 19.40 & 19.59 & 20.08 & 20.96 & 22.15 & 23.76 & 26.01 & 28.62 & 31.04 & 32.70 & 33.61 & 33.99 & 34.28 & 34.51 & 34.69 & 34.80 & 34.91 & 35.00 \\
\hline 9.30 & 9.37 & 9.44 & 9.48 & 9.51 & 9.50 & 9.50 & 9.49 & 9.47 & 9.42 & 9.37 & 9.32 & 9.25 & 9.20 & 9.14 & 9.05 & 8.98 & 8.94 & 8.88 & 8.81 & 8.74 & 8.71 \\
\hline 19.24 & 19.36 & 19.46 & 19.50 & 19.49 & 19.44 & 19.37 & 19.26 & 19.14 & 18.97 & 18.78 & 18.55 & 18.30 & 18.08 & 17.89 & 17.66 & 17.40 & 17.19 & 16.98 & 16.73 & 16.54 & 16.36 \\
\hline 12.21 & 12.22 & 12.25 & 12.28 & 12.26 & 12.27 & 12.33 & 12.38 & 12.43 & 12.43 & 12.43 & 12.36 & 12.24 & 12.13 & 12.06 & 11.98 & 11.85 & 11.72 & 11.62 & 11.52 & 11.41 & 11.31 \\
\hline 12.05 & 12.10 & 12.17 & 12.19 & 12.20 & 12.25 & 12.37 & 12.49 & 12.62 & 12.67 & 12.69 & 12.63 & 12.55 & 12.42 & 12.29 & 12.16 & 12.00 & 11.84 & 11.72 & 11.63 & 11.49 & 11.36 \\
\hline 14.89 & 14.93 & 15.00 & 15.05 & 15.07 & 15.11 & 15.15 & 15.26 & 15.36 & 15.37 & 15.35 & 15.31 & 15.25 & 15.16 & 15.04 & 14.93 & 14.82 & 14.70 & 14.60 & 14.51 & 14.44 & 14.32 \\
\hline 12.50 & 12.56 & 12.61 & 12.63 & 12.66 & 12.69 & 12.76 & 12.84 & 12.91 & 12.97 & 12.97 & 12.89 & 12.80 & 12.74 & 12.65 & 12.50 & 12.41 & 12.32 & 12.24 & 12.13 & 12.00 & 11.91 \\
\hline
\end{tabular}

Note: Only a portion of this table appears here. The complete table is available in ASCII format. 
Table T9. Spectral reflectance data, Hole 1098A.

\begin{tabular}{|c|c|c|c|c|c|c|c|c|c|c|c|c|c|c|c|c|c|c|c|c|c|}
\hline Core & $\begin{array}{l}\text { Core } \\
\text { type }\end{array}$ & Section & $\begin{array}{l}\text { Interval } \\
\text { top } \\
(\mathrm{cm})\end{array}$ & $\begin{array}{l}\text { Interval } \\
\text { bottom } \\
(\mathrm{cm})\end{array}$ & $\begin{array}{c}\text { Curator } \\
\text { depth } \\
\text { (mbsf) }\end{array}$ & $\begin{array}{l}\text { Recovery- } \\
\text { corrected } \\
\text { depth } \\
\text { (mbsf) }\end{array}$ & $L^{*}$ & $a^{*}$ & $b^{*}$ & Munsell & Munsell & Munsell & 400 & 410 & 420 & 430 & 440 & 450 & 460 & 470 & 480 \\
\hline 1 & $\mathrm{H}$ & 1 & 1 & 1.1 & 0.01 & 0.01 & 30.54 & 0.51 & 3.29 & $7.2 \mathrm{Y}$ & $3.0 /$ & 0.5 & 5.27 & 5.26 & 5.29 & 5.35 & 5.45 & 5.56 & 5.67 & 5.79 & 5.91 \\
\hline 1 & $\mathrm{H}$ & 1 & 3 & 3.1 & 0.03 & 0.03 & 33.49 & 0.57 & 4.62 & $7.6 \mathrm{Y}$ & 3.21 & 0.7 & 5.98 & 5.93 & 5.96 & 6.05 & 6.18 & 6.34 & 6.51 & 6.71 & 6.91 \\
\hline 1 & $\mathrm{H}$ & 1 & 5 & 5.1 & 0.05 & 0.05 & 38.88 & 0.24 & 6.82 & $9.3 \mathrm{Y}$ & $3.8 /$ & 1.1 & 7.14 & 7.09 & 7.14 & 7.32 & 7.62 & 8.00 & 8.40 & 8.79 & 9.18 \\
\hline 1 & $\mathrm{H}$ & 1 & 7 & 7.1 & 0.07 & 0.07 & 42.22 & 1.03 & 10.68 & $8.0 \mathrm{Y}$ & $4.1 /$ & 1.6 & 7.09 & 7.03 & 7.11 & 7.37 & 7.78 & 8.29 & 8.86 & 9.48 & 10.10 \\
\hline 1 & $\mathrm{H}$ & 1 & 9 & 9.1 & 0.09 & 0.09 & 40.24 & 0.99 & 10.90 & $8.2 \mathrm{Y}$ & $3.9 /$ & 1.6 & 6.05 & 6.02 & 6.09 & 6.35 & 6.77 & 7.29 & 7.84 & 8.41 & 9.00 \\
\hline 1 & $\mathrm{H}$ & 1 & 11 & 11.1 & 0.11 & 0.11 & 42.14 & 1.37 & 12.59 & $7.7 Y$ & $4.0 /$ & 1.8 & 6.18 & 6.13 & 6.23 & 6.54 & 7.02 & 7.59 & 8.24 & 8.94 & 9.62 \\
\hline 1 & $\mathrm{H}$ & 1 & 13 & 13.1 & 0.13 & 0.13 & 43.27 & 1.55 & 12.78 & $7.4 \mathrm{Y}$ & $4.1 /$ & 1.8 & 6.69 & 6.63 & 6.72 & 7.02 & 7.50 & 8.10 & 8.76 & 9.46 & 10.15 \\
\hline 1 & $\mathrm{H}$ & 1 & 15 & 15.1 & 0.15 & 0.15 & 43.09 & 1.57 & 13.97 & $7.5 \mathrm{Y}$ & $4.1 /$ & 2 & 6.23 & 6.14 & 6.21 & 6.51 & 7.00 & 7.61 & 8.28 & 9.01 & 9.74 \\
\hline 1 & $\mathrm{H}$ & 1 & 17 & 17.1 & 0.17 & 0.17 & 41.36 & 1.76 & 14.89 & $7.3 Y$ & $4.0 /$ & 2.1 & 5.21 & 5.13 & 5.20 & 5.50 & 5.97 & 6.56 & 7.21 & 7.91 & 8.62 \\
\hline 1 & $\mathrm{H}$ & 1 & 19 & 19.1 & 0.19 & 0.19 & 43.83 & 1.72 & 14.10 & $7.2 \mathrm{Y}$ & $4.2 /$ & 2 & 6.57 & 6.48 & 6.58 & 6.88 & 7.35 & 7.94 & 8.61 & 9.34 & 10.07 \\
\hline 1 & $\mathrm{H}$ & 1 & 21 & 21.1 & 0.21 & 0.21 & 41.47 & 1.32 & 13.56 & $7.8 \mathrm{Y}$ & $4.0 /$ & 2 & 5.68 & 5.58 & 5.66 & 5.96 & 6.44 & 7.01 & 7.63 & 8.31 & 9.01 \\
\hline 1 & $\mathrm{H}$ & 1 & 23 & 23.1 & 0.23 & 0.23 & 40.54 & 0.64 & 10.70 & $8.8 \mathrm{Y}$ & $3.9 /$ & 1.6 & 6.16 & 6.12 & 6.20 & 6.46 & 6.91 & 7.48 & 8.07 & 8.66 & 9.24 \\
\hline 1 & $\mathrm{H}$ & 1 & 25 & 25.1 & 0.25 & 0.25 & 41.16 & 0.75 & 9.85 & $8.3 \mathrm{Y}$ & $4.0 /$ & 1.5 & 6.99 & 6.94 & 7.00 & 7.25 & 7.65 & 8.10 & 8.59 & 9.12 & 9.65 \\
\hline 1 & $\mathrm{H}$ & 1 & 27 & 27.1 & 0.27 & 0.27 & 44.49 & 1.02 & 8.68 & $7.2 Y$ & $4.3 /$ & 1.2 & 9.55 & 9.43 & 9.45 & 9.64 & 9.95 & 10.35 & 10.77 & 11.24 & 11.73 \\
\hline
\end{tabular}

\begin{tabular}{|c|c|c|c|c|c|c|c|c|c|c|c|c|c|c|c|c|c|c|c|c|c|}
\hline 490 & 500 & 510 & 520 & 530 & 540 & 550 & 560 & 570 & 580 & 590 & 600 & 610 & 620 & 630 & 640 & 650 & 660 & 670 & 680 & 690 & 700 \\
\hline 6.03 & 6.11 & 6.18 & 6.26 & 6.32 & 6.38 & 6.42 & 6.47 & 6.48 & 6.53 & 6.56 & 6.57 & 6.56 & 6.60 & 6.64 & 6.62 & 6.60 & 6.61 & 6.64 & 6.66 & 6.68 & 6.75 \\
\hline 7.09 & 7.23 & 7.37 & 7.45 & 7.56 & 7.65 & 7.72 & 7.78 & 7.84 & 7.88 & 7.92 & 7.91 & 7.91 & 7.95 & 7.97 & 7.97 & 7.98 & 7.97 & 7.95 & 7.98 & 8.07 & 8.17 \\
\hline 9.53 & 9.81 & 10.06 & 10.23 & 10.34 & 10.46 & 10.56 & 10.66 & 10.73 & 10.79 & 10.81 & 10.79 & 10.77 & 10.75 & 10.80 & 10.77 & 10.76 & 10.74 & 10.70 & 10.73 & 10.82 & 10.93 \\
\hline 10.64 & 11.10 & 11.51 & 11.81 & 12.09 & 12.30 & 12.49 & 12.71 & 12.88 & 13.02 & 13.06 & 13.06 & 13.08 & 13.14 & 13.23 & 13.22 & 13.20 & 13.14 & 13.10 & 13.20 & 13.37 & 13.52 \\
\hline 9.53 & 9.97 & 10.33 & 10.61 & 10.88 & 11.08 & 11.29 & 11.48 & 11.59 & 11.75 & 11.78 & 11.78 & 11.81 & 11.85 & 11.93 & 11.95 & 11.91 & 11.82 & 11.78 & 11.90 & 12.06 & 12.21 \\
\hline 10.25 & 10.78 & 11.23 & 11.58 & 11.90 & 12.17 & 12.42 & 12.64 & 12.83 & 13.02 & 13.10 & 13.11 & 13.13 & 13.21 & 13.33 & 13.33 & 13.31 & 13.22 & 13.17 & 13.27 & 13.47 & 13.71 \\
\hline 10.79 & 11.34 & 11.83 & 12.22 & 12.60 & 12.88 & 13.14 & 13.39 & 13.61 & 13.79 & 13.88 & 13.92 & 13.96 & 14.04 & 14.16 & 14.20 & 14.18 & 14.05 & 13.97 & 14.10 & 14.36 & 14.63 \\
\hline 10.43 & 11.03 & 11.57 & 11.99 & 12.43 & 12.76 & 13.04 & 13.30 & 13.53 & 13.71 & 13.82 & 13.80 & 13.82 & 13.91 & 14.08 & 14.13 & 14.06 & 13.85 & 13.71 & 13.90 & 14.21 & 14.51 \\
\hline 9.26 & 9.83 & 10.37 & 10.81 & 11.28 & 11.61 & 11.92 & 12.18 & 12.41 & 12.60 & 12.70 & 12.69 & 12.74 & 12.83 & 12.96 & 12.99 & 12.89 & 12.65 & 12.50 & 12.69 & 13.05 & 13.42 \\
\hline 10.75 & 11.35 & 11.92 & 12.40 & 12.85 & 13.21 & 13.50 & 13.82 & 14.07 & 14.29 & 14.38 & 14.39 & 14.42 & 14.48 & 14.62 & 14.68 & 14.57 & 14.32 & 14.16 & 14.30 & 14.64 & 14.96 \\
\hline 9.62 & 10.17 & 10.68 & 11.08 & 11.48 & 11.79 & 12.03 & 12.28 & 12.48 & 12.61 & 12.69 & 12.68 & 12.70 & 12.78 & 12.90 & 12.90 & 12.80 & 12.56 & 12.41 & 12.57 & 12.91 & 13.21 \\
\hline 9.79 & 10.22 & 10.59 & 10.91 & 11.17 & 11.35 & 11.53 & 11.69 & 11.78 & 11.90 & 11.94 & 11.92 & 11.93 & 11.97 & 12.03 & 12.01 & 11.96 & 11.85 & 11.78 & 11.89 & 12.08 & 12.31 \\
\hline 10.13 & 10.52 & 10.89 & 11.21 & 11.52 & 11.73 & 11.90 & 12.08 & 12.23 & 12.28 & 12.32 & 12.30 & 12.32 & 12.35 & 12.42 & 12.43 & 12.36 & 12.20 & 12.08 & 12.18 & 12.43 & 12.71 \\
\hline 12.19 & 12.59 & 12.97 & 13.31 & 13.62 & 13.84 & 14.06 & 14.28 & 14.43 & 14.58 & 14.62 & 14.60 & 14.60 & 14.67 & 14.75 & 14.74 & 14.69 & 14.48 & 14.31 & 14.42 & 14.72 & 14.99 \\
\hline
\end{tabular}

Note: Only a portion of this table appears here. The complete table is available in ASCII format. 
Table T10. Spectral reflectance data, Hole 1098B.

\begin{tabular}{|c|c|c|c|c|c|c|c|c|c|c|c|c|c|c|c|c|c|c|c|c|c|}
\hline Core & $\begin{array}{l}\text { Core } \\
\text { type }\end{array}$ & Section & $\begin{array}{l}\text { Interval } \\
\text { top } \\
(\mathrm{cm})\end{array}$ & $\begin{array}{l}\text { Interval } \\
\text { bottom } \\
(\mathrm{cm})\end{array}$ & $\begin{array}{c}\text { Curator } \\
\text { depth } \\
\text { (mbsf) }\end{array}$ & $\begin{array}{l}\text { Recovery- } \\
\text { corrected } \\
\text { depth } \\
\text { (mbsf) }\end{array}$ & $L^{*}$ & $a^{*}$ & $b^{*}$ & Munsell & Munsell & Munsell & 400 & 410 & 420 & 430 & 440 & 450 & 460 & 470 & 480 \\
\hline 1 & $\mathrm{H}$ & 1 & 1 & 1.1 & 0.01 & 0.01 & 37.49 & 0.26 & 6.45 & $9.1 \mathrm{Y}$ & $3.6 /$ & 1 & 6.77 & 6.70 & 6.75 & 6.91 & 7.17 & 7.49 & 7.82 & 8.16 & 8.49 \\
\hline 1 & $\mathrm{H}$ & 1 & 2 & 2.1 & 0.02 & 0.02 & 40.65 & 0.85 & 10.04 & $8.2 \mathrm{Y}$ & $3.9 /$ & 1.5 & 6.61 & 6.57 & 6.64 & 6.89 & 7.29 & 7.76 & 8.30 & 8.85 & 9.37 \\
\hline 1 & $\mathrm{H}$ & 1 & 3 & 3.1 & 0.03 & 0.03 & 39.03 & 0.92 & 9.40 & $8.1 \mathrm{Y}$ & $3.8 /$ & 1.4 & 6.18 & 6.14 & 6.24 & 6.48 & 6.81 & 7.23 & 7.71 & 8.19 & 8.67 \\
\hline 1 & $\mathrm{H}$ & 1 & 4 & 4.1 & 0.04 & 0.04 & 42.34 & 0.82 & 7.20 & $7.7 \mathrm{Y}$ & $4.1 /$ & 1.1 & 8.93 & 8.87 & 8.87 & 9.04 & 9.35 & 9.70 & 10.08 & 10.48 & 10.89 \\
\hline 1 & $\mathrm{H}$ & 1 & 5 & 5.1 & 0.05 & 0.05 & 42.22 & 1.30 & 11.99 & $7.7 Y$ & $4.1 /$ & 1.7 & 6.52 & 6.47 & 6.54 & 6.84 & 7.30 & 7.86 & 8.47 & 9.12 & 9.77 \\
\hline 1 & $\mathrm{H}$ & 1 & 6 & 6.1 & 0.06 & 0.06 & 42.95 & 1.25 & 11.04 & $7.6 \mathrm{Y}$ & $4.1 /$ & 1.6 & 7.31 & 7.24 & 7.31 & 7.59 & 8.03 & 8.55 & 9.15 & 9.77 & 10.38 \\
\hline 1 & $\mathrm{H}$ & 1 & 7 & 7.1 & 0.07 & 0.07 & 41.04 & 1.53 & 13.38 & $7.6 \mathrm{Y}$ & $3.9 /$ & 1.9 & 5.50 & 5.42 & 5.49 & 5.81 & 6.30 & 6.86 & 7.49 & 8.17 & 8.85 \\
\hline 1 & $\mathrm{H}$ & 1 & 8 & 8.1 & 0.08 & 0.08 & 44.32 & 1.24 & 12.32 & $7.9 \mathrm{Y}$ & $4.3 /$ & 1.8 & 7.32 & 7.21 & 7.28 & 7.61 & 8.13 & 8.76 & 9.46 & 10.19 & 10.91 \\
\hline 1 & $\mathrm{H}$ & 1 & 9 & 9.1 & 0.09 & 0.09 & 44.60 & 1.19 & 11.47 & $7.8 \mathrm{Y}$ & $4.3 /$ & 1.7 & 7.85 & 7.77 & 7.85 & 8.15 & 8.66 & 9.25 & 9.90 & 10.58 & 11.26 \\
\hline 1 & $\mathrm{H}$ & 1 & 10 & 10.1 & 0.10 & 0.10 & 42.75 & 1.40 & 12.42 & $7.5 \mathrm{Y}$ & $4.1 /$ & 1.8 & 6.74 & 6.67 & 6.74 & 7.02 & 7.47 & 8.00 & 8.58 & 9.23 & 9.88 \\
\hline 1 & $\mathrm{H}$ & 1 & 11 & 11.1 & 0.11 & 0.11 & 39.72 & 1.36 & 12.64 & $7.6 \mathrm{Y}$ & $3.8 /$ & 1.9 & 5.47 & 5.37 & 5.44 & 5.70 & 6.09 & 6.56 & 7.09 & 7.66 & 8.24 \\
\hline 1 & $\mathrm{H}$ & 1 & 12 & 12.1 & 0.12 & 0.12 & 38.92 & 1.05 & 11.18 & $8.0 \mathrm{Y}$ & $3.7 /$ & 1.7 & 5.59 & 5.50 & 5.53 & 5.80 & 6.18 & 6.64 & 7.14 & 7.68 & 8.23 \\
\hline 1 & $\mathrm{H}$ & 1 & 13 & 13.1 & 0.13 & 0.13 & 39.60 & 1.10 & 12.30 & $8.0 \mathrm{Y}$ & $3.8 /$ & 1.8 & 5.37 & 5.29 & 5.35 & 5.63 & 6.08 & 6.62 & 7.17 & 7.73 & 8.31 \\
\hline 1 & $\mathrm{H}$ & 1 & 14 & 14.1 & 0.14 & 0.14 & 41.06 & 1.70 & 13.61 & $7.0 \mathrm{Y}$ & $3.9 /$ & 1.9 & 5.75 & 5.65 & 5.69 & 5.95 & 6.39 & 6.90 & 7.45 & 8.03 & 8.64 \\
\hline
\end{tabular}

\begin{tabular}{|c|c|c|c|c|c|c|c|c|c|c|c|c|c|c|c|c|c|c|c|c|c|}
\hline 490 & 500 & 510 & 520 & 530 & 540 & 550 & 560 & 570 & 580 & 590 & 600 & 610 & 620 & 630 & 640 & 650 & 660 & 670 & 680 & 690 & 700 \\
\hline 8.80 & 9.09 & 9.32 & 9.45 & 9.57 & 9.68 & 9.80 & 9.89 & 9.93 & 9.98 & 9.98 & 9.97 & 9.98 & 9.97 & 10.01 & 9.99 & 9.96 & 9.93 & 9.92 & 9.99 & 10.06 & 10.16 \\
\hline 9.85 & 10.28 & 10.65 & 10.93 & 11.19 & 11.37 & 11.57 & 11.72 & 11.85 & 11.99 & 12.01 & 11.99 & 12.01 & 12.05 & 12.14 & 12.14 & 12.11 & 12.04 & 12.01 & 12.08 & 12.22 & 12.41 \\
\hline 9.09 & 9.47 & 9.80 & 10.02 & 10.24 & 10.42 & 10.57 & 10.74 & 10.85 & 10.95 & 11.02 & 11.00 & 11.03 & 11.07 & 11.15 & 11.14 & 11.15 & 11.10 & 11.07 & 11.16 & 11.26 & 11.39 \\
\hline 11.27 & 11.60 & 11.89 & 12.11 & 12.29 & 12.45 & 12.64 & 12.78 & 12.90 & 12.98 & 13.03 & 13.01 & 13.03 & 13.09 & 13.18 & 13.17 & 13.16 & 13.12 & 13.10 & 13.21 & 13.31 & 13.40 \\
\hline 10.36 & 10.86 & 11.32 & 11.67 & 12.00 & 12.23 & 12.49 & 12.71 & 12.89 & 13.05 & 13.12 & 13.11 & 13.16 & 13.22 & 13.35 & 13.39 & 13.33 & 13.21 & 13.19 & 13.32 & 13.51 & 13.74 \\
\hline 10.93 & 11.43 & 11.87 & 12.19 & 12.48 & 12.73 & 12.97 & 13.20 & 13.37 & 13.51 & 13.59 & 13.57 & 13.62 & 13.70 & 13.81 & 13.83 & 13.82 & 13.74 & 13.68 & 13.77 & 13.95 & 14.20 \\
\hline 9.45 & 9.98 & 10.46 & 10.83 & 11.18 & 11.45 & 11.72 & 11.96 & 12.16 & 12.31 & 12.39 & 12.42 & 12.45 & 12.53 & 12.68 & 12.68 & 12.64 & 12.55 & 12.50 & 12.62 & 12.84 & 13.09 \\
\hline 11.56 & 12.13 & 12.63 & 13.01 & 13.36 & 13.64 & 13.92 & 14.15 & 14.33 & 14.50 & 14.57 & 14.56 & 14.61 & 14.68 & 14.83 & 14.84 & 14.79 & 14.65 & 14.54 & 14.68 & 14.95 & 15.23 \\
\hline 11.87 & 12.39 & 12.88 & 13.25 & 13.59 & 13.86 & 14.12 & 14.35 & 14.52 & 14.68 & 14.75 & 14.73 & 14.78 & 14.87 & 15.00 & 15.00 & 14.95 & 14.80 & 14.71 & 14.86 & 15.09 & 15.34 \\
\hline 10.49 & 11.02 & 11.50 & 11.89 & 12.27 & 12.57 & 12.87 & 13.09 & 13.29 & 13.43 & 13.53 & 13.50 & 13.55 & 13.64 & 13.75 & 13.75 & 13.69 & 13.53 & 13.44 & 13.57 & 13.81 & 14.08 \\
\hline 8.78 & 9.27 & 9.73 & 10.08 & 10.45 & 10.72 & 10.96 & 11.19 & 11.38 & 11.48 & 11.55 & 11.56 & 11.59 & 11.63 & 11.74 & 11.75 & 11.70 & 11.54 & 11.43 & 11.56 & 11.79 & 12.07 \\
\hline 8.71 & 9.13 & 9.52 & 9.81 & 10.10 & 10.33 & 10.55 & 10.71 & 10.82 & 10.94 & 11.00 & 11.00 & 11.01 & 11.06 & 11.16 & 11.16 & 11.10 & 10.99 & 10.96 & 11.08 & 11.28 & 11.49 \\
\hline 8.85 & 9.29 & 9.72 & 10.09 & 10.43 & 10.69 & 10.94 & 11.16 & 11.29 & 11.41 & 11.44 & 11.42 & 11.44 & 11.51 & 11.59 & 11.60 & 11.52 & 11.30 & 11.19 & 11.37 & 11.66 & 11.96 \\
\hline 9.20 & 9.69 & 10.16 & 10.63 & 11.06 & 11.44 & 11.78 & 12.06 & 12.27 & 12.43 & 12.51 & 12.51 & 12.53 & 12.58 & 12.68 & 12.70 & 12.58 & 12.26 & 12.06 & 12.24 & 12.65 & 13.07 \\
\hline
\end{tabular}

Note: Only a portion of this table appears here. The complete table is available in ASCII format. 
Table T11. Spectral reflectance data, Hole 1098C.

\begin{tabular}{|c|c|c|c|c|c|c|c|c|c|c|c|c|c|c|c|c|c|c|c|c|c|}
\hline Core & $\begin{array}{l}\text { Core } \\
\text { type }\end{array}$ & Section & $\begin{array}{c}\text { Interval } \\
\text { top } \\
(\mathrm{cm})\end{array}$ & $\begin{array}{l}\text { Interval } \\
\text { bottom } \\
(\mathrm{cm})\end{array}$ & $\begin{array}{l}\text { Curator } \\
\text { depth } \\
\text { (mbsf) }\end{array}$ & $\begin{array}{l}\text { Recovery- } \\
\text { corrected } \\
\text { depth } \\
\text { (mbsf) }\end{array}$ & $\mathrm{L}^{*}$ & $a^{*}$ & $b^{*}$ & Munsell & Munsell & Munsell & 400 & 410 & 420 & 430 & 440 & 450 & 460 & 470 & 480 \\
\hline 1 & $\mathrm{H}$ & 1 & 1 & 1.1 & 0.01 & 0.01 & 38.37 & 0.30 & 7.46 & $9.1 \mathrm{Y}$ & $3.7 /$ & 1.2 & 6.70 & 6.60 & 6.65 & 6.87 & 7.17 & 7.53 & 7.94 & 8.38 & 8.78 \\
\hline 1 & $\mathrm{H}$ & 1 & 3 & 3.1 & 0.03 & 0.03 & 39.16 & 0.57 & 8.22 & $8.6 \mathrm{Y}$ & $3.8 /$ & 1.3 & 6.78 & 6.72 & 6.76 & 6.97 & 7.28 & 7.69 & 8.11 & 8.54 & 8.97 \\
\hline 1 & $\mathrm{H}$ & 1 & 5 & 5.1 & 0.05 & 0.05 & 39.53 & 0.71 & 9.37 & $8.5 Y$ & $3.8 /$ & 1.4 & 6.41 & 6.34 & 6.41 & 6.64 & 7.00 & 7.45 & 7.93 & 8.46 & 8.97 \\
\hline 1 & $\mathrm{H}$ & 1 & 7 & 7.1 & 0.07 & 0.07 & 41.95 & 1.16 & 11.14 & $7.9 \mathrm{Y}$ & $4.0 /$ & 1.6 & 6.73 & 6.68 & 6.75 & 7.02 & 7.47 & 8.01 & 8.60 & 9.23 & 9.84 \\
\hline 1 & $\mathrm{H}$ & 1 & 9 & 9.1 & 0.09 & 0.09 & 43.76 & 1.42 & 12.00 & $7.5 \mathrm{Y}$ & 4.21 & 1.7 & 7.29 & 7.20 & 7.28 & 7.57 & 8.03 & 8.60 & 9.25 & 9.94 & 10.62 \\
\hline 1 & $\mathrm{H}$ & 1 & 11 & 11.1 & 0.11 & 0.11 & 45.68 & 1.34 & 12.22 & $7.6 \mathrm{Y}$ & $4.4 /$ & 1.8 & 8.08 & 8.00 & 8.09 & 8.41 & 8.93 & 9.55 & 10.23 & 10.97 & 11.70 \\
\hline 1 & $\mathrm{H}$ & 1 & 13 & 13.1 & 0.13 & 0.13 & 44.62 & 1.50 & 13.02 & $7.5 Y$ & $4.3 /$ & 1.9 & 7.27 & 7.16 & 7.23 & 7.55 & 8.08 & 8.71 & 9.38 & 10.12 & 10.86 \\
\hline 1 & $\mathrm{H}$ & 1 & 15 & 15.1 & 0.15 & 0.15 & 46.01 & 1.43 & 11.39 & $7.0 \mathrm{Y}$ & $4.4 /$ & 1.6 & 8.76 & 8.68 & 8.76 & 9.06 & 9.53 & 10.12 & 10.73 & 11.38 & 12.03 \\
\hline 1 & $\mathrm{H}$ & 1 & 17 & 17.1 & 0.17 & 0.17 & 42.54 & 1.06 & 11.10 & $7.9 \mathrm{Y}$ & $4.1 /$ & 1.6 & 7.06 & 7.00 & 7.07 & 7.38 & 7.81 & 8.32 & 8.91 & 9.53 & 10.13 \\
\hline 1 & $\mathrm{H}$ & 1 & 19 & 19.1 & 0.19 & 0.19 & 43.35 & 1.51 & 12.76 & $7.2 Y$ & 4.21 & 1.8 & 6.95 & 6.87 & 6.94 & 7.23 & 7.67 & 8.22 & 8.81 & 9.43 & 10.06 \\
\hline 1 & $\mathrm{H}$ & 1 & 21 & 21.1 & 0.21 & 0.21 & 39.18 & 0.36 & 7.58 & $9.0 \mathrm{Y}$ & $3.8 /$ & 1.2 & 6.94 & 6.86 & 6.94 & 7.17 & 7.50 & 7.90 & 8.32 & 8.75 & 9.16 \\
\hline 1 & $\mathrm{H}$ & 1 & 23 & 23.1 & 0.23 & 0.23 & 43.67 & 1.16 & 10.83 & $7.6 \mathrm{Y}$ & 4.21 & 1.6 & 7.80 & 7.73 & 7.81 & 8.08 & 8.51 & 9.05 & 9.61 & 10.20 & 10.78 \\
\hline 1 & $\mathrm{H}$ & 1 & 25 & 25.1 & 0.25 & 0.25 & 45.90 & 1.11 & 11.52 & $7.8 \mathrm{Y}$ & $4.4 /$ & 1.7 & 8.60 & 8.50 & 8.56 & 8.86 & 9.35 & 9.95 & 10.61 & 11.30 & 11.99 \\
\hline 1 & $\mathrm{H}$ & 1 & 27 & 27.1 & 0.27 & 0.27 & 43.59 & 1.40 & 11.91 & $7.3 Y$ & 4.21 & 1.7 & 7.34 & 7.30 & 7.38 & 7.65 & 8.09 & 8.63 & 9.20 & 9.80 & 10.42 \\
\hline
\end{tabular}

\begin{tabular}{rrrrrrcccccccccccccccc}
\hline \multicolumn{1}{c}{490} & 500 & 510 & 520 & 530 & 540 & 550 & 560 & 570 & 580 & 590 & 600 & 610 & 620 & 630 & 640 & 650 & 660 & 670 & 680 & 690 & 700 \\
\hline 9.36 & 9.71 & 10.03 & 10.21 & 10.42 & 10.58 & 10.68 & 10.83 & 10.92 & 11.00 & 11.04 & 11.01 & 11.02 & 11.03 & 11.10 & 11.09 & 11.08 & 11.03 & 10.99 & 11.05 & 11.15 & 11.31 \\
9.42 & 9.80 & 10.13 & 10.35 & 10.57 & 10.75 & 10.89 & 11.01 & 11.14 & 11.27 & 11.31 & 11.27 & 11.26 & 11.29 & 11.38 & 11.41 & 11.37 & 11.31 & 11.30 & 11.40 & 11.54 & 11.67 \\
10.42 & 10.89 & 11.31 & 11.62 & 11.88 & 12.10 & 12.32 & 12.52 & 12.67 & 12.84 & 12.92 & 12.92 & 12.94 & 13.01 & 13.11 & 13.11 & 13.10 & 13.04 & 12.98 & 13.08 & 13.29 & 13.52 \\
11.23 & 11.77 & 12.25 & 12.64 & 12.96 & 13.24 & 13.48 & 13.71 & 13.91 & 14.11 & 14.19 & 14.22 & 14.25 & 14.32 & 14.44 & 14.49 & 14.46 & 14.34 & 14.26 & 14.38 & 14.62 & 14.89 \\
12.37 & 12.94 & 13.46 & 13.89 & 14.29 & 14.60 & 14.87 & 15.10 & 15.32 & 15.51 & 15.60 & 15.58 & 15.62 & 15.73 & 15.85 & 15.87 & 15.83 & 15.66 & 15.53 & 15.66 & 15.96 & 16.28 \\
11.52 & 12.10 & 12.62 & 13.05 & 13.50 & 13.80 & 14.09 & 14.36 & 14.60 & 14.77 & 14.86 & 14.85 & 14.89 & 14.99 & 15.14 & 15.18 & 15.10 & 14.89 & 14.74 & 14.89 & 15.25 & 15.59 \\
12.64 & 13.16 & 13.66 & 14.05 & 14.46 & 14.81 & 15.10 & 15.37 & 15.58 & 15.78 & 15.87 & 15.89 & 15.90 & 15.96 & 16.08 & 16.09 & 16.03 & 15.76 & 15.55 & 15.73 & 16.12 & 16.49 \\
10.69 & 11.15 & 11.56 & 11.93 & 12.30 & 12.53 & 12.75 & 12.94 & 13.10 & 13.23 & 13.30 & 13.29 & 13.31 & 13.37 & 13.45 & 13.49 & 13.41 & 13.22 & 13.10 & 13.25 & 13.54 & 13.81 \\
10.66 & 11.19 & 11.70 & 12.16 & 12.61 & 12.96 & 13.25 & 13.53 & 13.73 & 13.92 & 13.99 & 14.00 & 14.00 & 14.06 & 14.18 & 14.19 & 14.09 & 13.81 & 13.59 & 13.78 & 14.21 & 14.59 \\
9.54 & 9.84 & 10.09 & 10.30 & 10.50 & 10.64 & 10.75 & 10.86 & 10.92 & 10.96 & 10.99 & 10.98 & 10.98 & 11.00 & 11.05 & 11.03 & 11.01 & 10.94 & 10.88 & 10.95 & 11.11 & 11.28 \\
11.32 & 11.80 & 12.25 & 12.63 & 13.00 & 13.25 & 13.49 & 13.71 & 13.89 & 14.03 & 14.08 & 14.08 & 14.10 & 14.16 & 14.25 & 14.27 & 14.25 & 14.10 & 13.98 & 14.09 & 14.39 & 14.69 \\
12.64 & 13.18 & 13.69 & 14.11 & 14.50 & 14.81 & 15.09 & 15.32 & 15.49 & 15.67 & 15.73 & 15.71 & 15.71 & 15.78 & 15.90 & 15.91 & 15.85 & 15.63 & 15.49 & 15.63 & 15.93 & 16.22 \\
11.02 & 11.52 & 12.00 & 12.43 & 12.82 & 13.12 & 13.41 & 13.67 & 13.86 & 14.03 & 14.12 & 14.11 & 14.11 & 14.16 & 14.30 & 14.33 & 14.23 & 14.00 & 13.85 & 14.02 & 14.38 & 14.71 \\
\hline
\end{tabular}

Note: Only a portion of this table appears here. The complete table is available in ASCII format. 
Table T12. Spectral reflectance data, Hole 1099A.

\begin{tabular}{|c|c|c|c|c|c|c|c|c|c|c|c|c|c|c|c|c|c|c|c|c|c|}
\hline Core & $\begin{array}{l}\text { Core } \\
\text { type }\end{array}$ & Section & $\begin{array}{c}\text { Interval } \\
\text { top } \\
(\mathrm{cm})\end{array}$ & $\begin{array}{l}\text { Interval } \\
\text { bottom } \\
(\mathrm{cm})\end{array}$ & $\begin{array}{l}\text { Curator } \\
\text { depth } \\
\text { (mbsf) }\end{array}$ & $\begin{array}{l}\text { Recovery- } \\
\text { corrected } \\
\text { depth } \\
\text { (mbsf) }\end{array}$ & $\mathrm{L}^{*}$ & $a^{*}$ & $b^{*}$ & Munsell & Munsell & Munsell & 400 & 410 & 420 & 430 & 440 & 450 & 460 & 470 & 480 \\
\hline 1 & $\mathrm{H}$ & 1 & 1 & 1.1 & 0.01 & 0.01 & 40.67 & 0.91 & 11.48 & $8.3 \mathrm{Y}$ & $3.9 /$ & 1.7 & 5.97 & 5.92 & 6.04 & 6.33 & 6.76 & 7.29 & 7.86 & 8.49 & 9.09 \\
\hline 1 & $\mathrm{H}$ & 1 & 3 & 3.1 & 0.03 & 0.03 & 43.75 & 1.14 & 10.90 & $7.8 \mathrm{Y}$ & 4.21 & 1.6 & 7.65 & 7.60 & 7.70 & 7.99 & 8.46 & 9.03 & 9.63 & 10.27 & 10.89 \\
\hline 1 & $\mathrm{H}$ & 1 & 5 & 5.1 & 0.05 & 0.05 & 42.86 & 1.17 & 10.94 & $7.7 Y$ & $4.1 /$ & 1.6 & 7.25 & 7.21 & 7.33 & 7.60 & 8.03 & 8.57 & 9.13 & 9.73 & 10.34 \\
\hline 1 & $\mathrm{H}$ & 1 & 7 & 7.1 & 0.07 & 0.07 & 41.86 & 0.92 & 9.92 & $7.9 \mathrm{Y}$ & $4.0 /$ & 1.4 & 7.28 & 7.22 & 7.31 & 7.57 & 7.95 & 8.43 & 8.95 & 9.49 & 10.01 \\
\hline 1 & $\mathrm{H}$ & 1 & 9 & 9.1 & 0.09 & 0.09 & 42.16 & 0.24 & 5.96 & $9.0 \mathrm{Y}$ & $4.1 /$ & 0.9 & 9.23 & 9.17 & 9.24 & 9.44 & 9.70 & 10.04 & 10.42 & 10.80 & 11.14 \\
\hline 1 & $\mathrm{H}$ & 1 & 11 & 11.1 & 0.11 & 0.11 & 45.48 & 0.41 & 8.28 & $8.9 \mathrm{Y}$ & $4.4 /$ & 1.2 & 9.72 & 9.69 & 9.80 & 10.09 & 10.50 & 11.02 & 11.55 & 12.11 & 12.65 \\
\hline 1 & $\mathrm{H}$ & 1 & 13 & 13.1 & 0.13 & 0.13 & 44.63 & -0.03 & 6.92 & $9.9 \mathrm{Y}$ & $4.3 /$ & 1.1 & 9.82 & 9.83 & 9.93 & 10.18 & 10.58 & 11.05 & 11.54 & 12.06 & 12.55 \\
\hline 1 & $\mathrm{H}$ & 1 & 15 & 15.1 & 0.15 & 0.15 & 40.79 & 0.48 & 9.43 & $8.8 \mathrm{Y}$ & $3.9 /$ & 1.4 & 6.84 & 6.80 & 6.90 & 7.16 & 7.57 & 8.05 & 8.55 & 9.08 & 9.61 \\
\hline 1 & $\mathrm{H}$ & 1 & 17 & 17.1 & 0.17 & 0.17 & 44.29 & 0.58 & 9.89 & $8.7 Y$ & $4.3 /$ & 1.5 & 8.20 & 8.18 & 8.31 & 8.62 & 9.09 & 9.66 & 10.27 & 10.91 & 11.53 \\
\hline 1 & $\mathrm{H}$ & 1 & 19 & 19.1 & 0.19 & 0.19 & 41.77 & 0.71 & 11.11 & $8.6 \mathrm{Y}$ & $4.0 /$ & 1.7 & 6.41 & 6.39 & 6.52 & 6.87 & 7.36 & 7.92 & 8.52 & 9.17 & 9.80 \\
\hline 1 & $\mathrm{H}$ & 1 & 21 & 21.1 & 0.21 & 0.21 & 43.22 & 0.66 & 10.59 & $8.6 \mathrm{Y}$ & 4.21 & 1.6 & 7.29 & 7.28 & 7.41 & 7.77 & 8.26 & 8.82 & 9.44 & 10.11 & 10.74 \\
\hline 1 & $\mathrm{H}$ & 1 & 23 & 23.1 & 0.23 & 0.23 & 43.11 & 0.42 & 10.48 & $9.1 \mathrm{Y}$ & $4.1 /$ & 1.6 & 7.24 & 7.24 & 7.37 & 7.71 & 8.21 & 8.79 & 9.42 & 10.09 & 10.73 \\
\hline 1 & $\mathrm{H}$ & 1 & 25 & 25.1 & 0.25 & 0.25 & 43.61 & 0.49 & 10.52 & $8.9 \mathrm{Y}$ & 4.21 & 1.6 & 7.51 & 7.50 & 7.62 & 7.97 & 8.46 & 9.04 & 9.68 & 10.34 & 10.98 \\
\hline 1 & $\mathrm{H}$ & 1 & 27 & 27.1 & 0.27 & 0.27 & 42.64 & 0.75 & 11.26 & $8.6 \mathrm{Y}$ & $4.1 /$ & 1.7 & 6.73 & 6.74 & 6.87 & 7.20 & 7.72 & 8.30 & 8.92 & 9.58 & 10.23 \\
\hline
\end{tabular}

\begin{tabular}{|c|c|c|c|c|c|c|c|c|c|c|c|c|c|c|c|c|c|c|c|c|c|}
\hline 490 & 500 & 510 & 520 & 530 & 540 & 550 & 560 & 570 & 580 & 590 & 600 & 610 & 620 & 630 & 640 & 650 & 660 & 670 & 680 & 690 & 700 \\
\hline 9.62 & 10.10 & 10.52 & 10.82 & 11.13 & 11.37 & 11.57 & 11.76 & 11.90 & 12.05 & 12.09 & 12.06 & 12.04 & 12.10 & 12.19 & 12.19 & 12.12 & 12.01 & 1.96 & 12.02 & 12.12 & 12.28 \\
\hline 11.47 & 11.97 & 12.41 & 12.74 & 13.05 & 13.28 & 13.50 & 13.75 & 13.91 & 14.07 & 14.15 & 14.14 & 14.16 & 14.22 & 14.32 & 14.36 & 14.31 & 14.19 & 14.14 & 14.23 & 14.40 & 14.60 \\
\hline 10.88 & 11.34 & 11.75 & 12.09 & 12.45 & 12.70 & 12.93 & 13.16 & 13.30 & 13.48 & 13.54 & 13.53 & 13.55 & 13.61 & 13.68 & 13.69 & 13.66 & 13.56 & 13.49 & 13.59 & 13.79 & 13.99 \\
\hline 10.48 & 10.89 & 11.29 & 11.61 & 11.91 & 12.14 & 12.31 & 12.52 & 12.66 & 12.76 & 12.79 & 12.78 & 12.79 & 12.86 & 12.92 & 12.94 & 12.93 & 12.83 & 12.74 & 12.83 & 13.03 & 13.23 \\
\hline 11.46 & 11.76 & 12.02 & 12.18 & 12.36 & 12.48 & 12.57 & 12.70 & 12.78 & 12.83 & 12.81 & 12.79 & 12.77 & 12.79 & 12.81 & 12.80 & 12.77 & 12.72 & 12.67 & 12.70 & 12.81 & 12.95 \\
\hline 13.16 & 13.58 & 13.95 & 14.25 & 14.51 & 14.67 & 14.86 & 15.01 & 15.11 & 15.21 & 15.24 & 15.20 & 15.18 & 15.21 & 15.25 & 15.22 & 15.15 & 15.08 & 15.02 & 15.07 & 15.16 & 15.27 \\
\hline 12.99 & 13.34 & 13.65 & 13.86 & 14.06 & 14.18 & 14.30 & 14.40 & 14.49 & 14.54 & 14.54 & 14.49 & 14.46 & 14.44 & 14.45 & 14.39 & 14.29 & 14.21 & 14.19 & 14.18 & 14.21 & 14.30 \\
\hline 10.07 & 10.46 & 10.83 & 11.09 & 11.33 & 11.54 & 11.72 & 11.86 & 11.96 & 12.05 & 12.07 & 12.05 & 12.02 & 12.01 & 12.09 & 12.07 & 11.99 & 11.90 & 11.86 & 11.89 & 12.00 & 12.16 \\
\hline 12.10 & 12.58 & 12.99 & 13.29 & 13.56 & 13.79 & 13.99 & 14.16 & 14.29 & 14.40 & 14.44 & 14.40 & 14.39 & 14.44 & 14.48 & 14.46 & 14.40 & 14.31 & 14.28 & 14.32 & 14.40 & 14.52 \\
\hline 10.37 & 10.84 & 11.25 & 11.57 & 11.86 & 12.10 & 12.28 & 12.46 & 12.61 & 12.73 & 12.76 & 12.72 & 12.71 & 12.77 & 12.83 & 12.80 & 12.75 & 12.67 & 12.61 & 12.68 & 12.78 & 12.91 \\
\hline 11.30 & 11.78 & 12.21 & 12.50 & 12.82 & 13.03 & 13.23 & 13.43 & 13.58 & 13.68 & 13.73 & 13.70 & 13.68 & 13.71 & 13.76 & 13.75 & 13.70 & 13.59 & 13.53 & 13.58 & 13.70 & 13.81 \\
\hline 11.30 & 11.79 & 12.21 & 12.53 & 12.80 & 13.01 & 13.20 & 13.38 & 13.51 & 13.61 & 13.62 & 13.57 & 13.56 & 13.59 & 13.62 & 13.57 & 13.50 & 13.40 & 13.35 & 13.37 & 13.43 & 13.50 \\
\hline 11.57 & 12.06 & 12.51 & 12.83 & 13.13 & 13.33 & 13.52 & 13.70 & 13.84 & 13.94 & 13.98 & 13.93 & 13.92 & 13.93 & 13.98 & 13.96 & 13.90 & 13.80 & 13.70 & 13.72 & 13.84 & 13.92 \\
\hline 10.83 & 11.32 & 11.76 & 12.10 & 12.41 & 12.62 & 12.84 & 13.03 & 13.18 & 13.32 & 13.36 & 13.32 & 13.31 & 13.34 & 13.43 & 13.43 & 13.34 & 13.22 & 13.17 & 13.25 & 13.40 & 13.49 \\
\hline
\end{tabular}

Note: Only a portion of this table appears here. The complete table is available in ASCII format. 
Table T13. Spectral reflectance data, Hole 1099B.

\begin{tabular}{|c|c|c|c|c|c|c|c|c|c|c|c|c|c|c|c|c|c|c|c|c|c|}
\hline Core & $\begin{array}{l}\text { Core } \\
\text { type }\end{array}$ & Section & $\begin{array}{l}\text { Interval } \\
\text { top } \\
(\mathrm{cm})\end{array}$ & $\begin{array}{l}\text { Interval } \\
\text { bottom } \\
(\mathrm{cm})\end{array}$ & $\begin{array}{l}\text { Curator } \\
\text { depth } \\
\text { (mbsf) }\end{array}$ & $\begin{array}{l}\text { Recovery- } \\
\text { corrected } \\
\text { depth } \\
\text { (mbsf) }\end{array}$ & $\mathrm{L}^{*}$ & $a^{*}$ & $b^{*}$ & Munsell & Munsell & Munsell & 400 & 410 & 420 & 430 & 440 & 450 & 460 & 470 & 480 \\
\hline 1 & $\mathrm{H}$ & 1 & 2 & 2.1 & 60.02 & 60.02 & 42.28 & -0.49 & 5.41 & $1.0 \mathrm{GY}$ & $4.1 /$ & 0.9 & 9.14 & 9.13 & 9.30 & 9.59 & 9.96 & 10.35 & 10.72 & 11.08 & 11.44 \\
\hline 1 & $\mathrm{H}$ & 1 & 7 & 7.1 & 60.07 & 60.07 & 46.53 & -0.50 & 4.00 & $1.5 \mathrm{GY}$ & $4.5 /$ & 0.7 & 12.76 & 12.67 & 12.74 & 12.97 & 13.28 & 13.62 & 13.95 & 14.29 & 14.60 \\
\hline 1 & $\mathrm{H}$ & 1 & 12 & 12.1 & 60.12 & 60.12 & 42.12 & -0.47 & 4.68 & $1.1 \mathrm{GY}$ & $4.1 /$ & 0.8 & 9.65 & 9.57 & 9.66 & 9.91 & 10.21 & 10.54 & 10.87 & 11.20 & 11.50 \\
\hline 1 & $\mathrm{H}$ & 1 & 17 & 17.1 & 60.17 & 60.16 & 46.63 & -0.39 & 3.20 & $1.5 \mathrm{GY}$ & $4.5 /$ & 0.5 & 13.32 & 13.27 & 13.35 & 13.55 & 13.80 & 14.09 & 14.36 & 14.64 & 14.88 \\
\hline 1 & $\mathrm{H}$ & 1 & 22 & 22.1 & 60.22 & 60.21 & 43.53 & -0.54 & 4.83 & $1.3 \mathrm{GY}$ & $4.2 /$ & 0.8 & 10.37 & 10.25 & 10.33 & 10.60 & 10.94 & 11.31 & 11.66 & 12.03 & 12.37 \\
\hline 1 & $\mathrm{H}$ & 1 & 27 & 27.1 & 60.27 & 60.26 & 38.87 & -0.58 & 6.30 & $1.0 \mathrm{GY}$ & $3.8 /$ & 1.1 & 7.10 & 7.05 & 7.15 & 7.44 & 7.82 & 8.21 & 8.57 & 8.95 & 9.32 \\
\hline 1 & $\mathrm{H}$ & 1 & 32 & 32.1 & 60.32 & 60.31 & 40.47 & -0.57 & 5.92 & $1.1 \mathrm{GY}$ & $3.9 /$ & 1 & 8.03 & 7.97 & 8.09 & 8.37 & 8.73 & 9.13 & 9.53 & 9.92 & 10.27 \\
\hline 1 & $\mathrm{H}$ & 1 & 37 & 37.1 & 60.37 & 60.36 & 40.30 & -0.58 & 6.35 & $1.0 \mathrm{GY}$ & $3.9 /$ & 1.1 & 7.71 & 7.63 & 7.75 & 8.07 & 8.48 & 8.89 & 9.30 & 9.71 & 10.09 \\
\hline 1 & $\mathrm{H}$ & 1 & 42 & 42.1 & 60.42 & 60.41 & 47.74 & -0.29 & 4.39 & $0.7 \mathrm{GY}$ & $4.6 /$ & 0.7 & 13.28 & 13.18 & 13.29 & 13.56 & 13.90 & 14.27 & 14.64 & 15.02 & 15.39 \\
\hline 1 & $\mathrm{H}$ & 1 & 47 & 47.1 & 60.47 & 60.46 & 50.27 & -0.12 & 1.84 & $0.7 \mathrm{GY}$ & $4.9 /$ & 0.3 & 17.06 & 17.01 & 17.11 & 17.26 & 17.40 & 17.57 & 17.73 & 17.92 & 18.06 \\
\hline 1 & $\mathrm{H}$ & 1 & 52 & 52.1 & 60.52 & 60.50 & 40.81 & -0.08 & 0.88 & $0.8 \mathrm{GY}$ & $4.0 /$ & 0.1 & 11.28 & 11.20 & 11.21 & 11.27 & 11.31 & 11.36 & 11.42 & 11.48 & 11.51 \\
\hline 1 & $\mathrm{H}$ & 1 & 57 & 57.1 & 60.57 & 60.55 & 38.16 & -0.06 & 1.33 & $0.2 \mathrm{GY}$ & $3.7 /$ & 0.2 & 9.50 & 9.45 & 9.47 & 9.54 & 9.58 & 9.65 & 9.74 & 9.81 & 9.88 \\
\hline 1 & $\mathrm{H}$ & 1 & 62 & 62.1 & 60.62 & 60.60 & 44.43 & 0.13 & 0.99 & $7.2 \mathrm{Y}$ & $4.3 /$ & 0.1 & 13.67 & 13.56 & 13.55 & 13.57 & 13.60 & 13.66 & 13.73 & 13.80 & 13.84 \\
\hline 1 & $\mathrm{H}$ & 1 & 67 & 67.1 & 60.67 & 60.65 & 47.18 & -0.31 & 3.48 & $1.0 \mathrm{GY}$ & $4.6 /$ & 0.6 & 13.63 & 13.54 & 13.62 & 13.83 & 14.06 & 14.34 & 14.62 & 14.91 & 15.18 \\
\hline
\end{tabular}

\begin{tabular}{|c|c|c|c|c|c|c|c|c|c|c|c|c|c|c|c|c|c|c|c|c|c|}
\hline 490 & 500 & 510 & 520 & 530 & 540 & 550 & 560 & 570 & 580 & 590 & 600 & 610 & 620 & 630 & 640 & 650 & 660 & 670 & 680 & 690 & 700 \\
\hline 11.76 & 12.01 & 12.25 & 12.45 & 12.60 & 12.73 & 12.83 & 12.86 & 12.87 & 12.90 & 12.83 & 12.76 & 12.69 & 12.67 & 12.65 & 12.57 & 12.5 & 12.41 & 12.35 & 12.36 & 12.39 & 12.45 \\
\hline 14.88 & 15.11 & 15.33 & 15.49 & 15.64 & 15.73 & 15.79 & 15.85 & 15.84 & 15.83 & 15.78 & 15.69 & 15.63 & 15.61 & 15.60 & 15.52 & 15.43 & 15.34 & 15.27 & 15.30 & 15.35 & 15.37 \\
\hline 11.77 & 11.97 & 12.20 & 12.41 & 12.53 & 12.62 & 12.71 & 12.75 & 12.73 & 12.75 & 12.69 & 12.64 & 12.59 & 12.55 & 12.53 & 12.46 & 12.39 & 12.33 & 12.28 & 12.26 & 12.30 & 12.38 \\
\hline 15.11 & 15.27 & 15.46 & 15.60 & 15.72 & 15.80 & 15.85 & 15.86 & 15.88 & 15.88 & 15.84 & 15.76 & 15.71 & 15.70 & 15.69 & 15.63 & 15.53 & 15.47 & 15.45 & 15.47 & 15.47 & 15.48 \\
\hline 12.67 & 12.89 & 13.12 & 13.32 & 13.47 & 13.60 & 13.67 & 13.69 & 13.70 & 13.71 & 13.66 & 13.57 & 13.51 & 13.47 & 13.45 & 13.36 & 13.30 & 13.21 & 13.14 & 13.15 & 13.22 & 13.27 \\
\hline 9.63 & 9.89 & 10.14 & 10.35 & 10.51 & 10.63 & 10.73 & 10.79 & 10.79 & 10.79 & 10.71 & 10.65 & 10.60 & 10.56 & 10.56 & 10.48 & 10.39 & 10.30 & 10.24 & 10.26 & 10.30 & 10.35 \\
\hline 10.60 & 10.86 & 11.11 & 11.32 & 11.49 & 11.58 & 11.66 & 11.71 & 11.74 & 11.74 & 11.69 & 11.63 & 11.53 & 11.50 & 11.48 & 11.42 & 11.34 & 11.23 & 11.17 & 11.18 & 11.23 & 11.30 \\
\hline 10.43 & 10.71 & 10.97 & 11.18 & 11.37 & 11.49 & 11.55 & 11.62 & 11.67 & 11.65 & 11.57 & 11.50 & 11.45 & 11.42 & 11.37 & 11.30 & 11.25 & 11.14 & 11.06 & 11.08 & 11.14 & 11.19 \\
\hline 15.70 & 15.92 & 16.15 & 16.32 & 16.50 & 16.61 & 16.69 & 16.76 & 16.77 & 16.78 & 16.74 & 16.68 & 16.63 & 16.62 & 16.64 & 16.53 & 16.44 & 16.40 & 16.37 & 16.38 & 16.43 & 16.49 \\
\hline 18.20 & 18.32 & 18.45 & 18.50 & 18.61 & 18.67 & 18.68 & 18.69 & 18.72 & 18.76 & 18.71 & 18.68 & 18.67 & 18.66 & 18.64 & 18.60 & 18.58 & 18.58 & 18.54 & 18.53 & 18.57 & 18.59 \\
\hline 11.59 & 11.62 & 11.66 & 11.70 & 11.74 & 11.75 & 11.77 & 11.78 & 11.75 & 11.79 & 11.77 & 11.75 & 11.74 & 11.74 & 11.76 & 11.73 & 11.71 & 11.70 & 11.68 & 11.67 & 11.70 & 11.71 \\
\hline 9.97 & 10.03 & 10.09 & 10.11 & 10.14 & 10.18 & 10.19 & 10.19 & 10.21 & 10.25 & 10.23 & 10.19 & 10.20 & 10.20 & 10.18 & 10.15 & 10.15 & 10.14 & 10.13 & 10.14 & 10.17 & 10.16 \\
\hline 13.90 & 13.94 & 13.99 & 14.04 & 14.09 & 14.11 & 14.10 & 14.15 & 14.17 & 14.20 & 14.19 & 14.17 & 14.17 & 14.20 & 14.23 & 14.21 & 14.21 & 14.22 & 14.21 & 14.21 & 14.23 & 14.26 \\
\hline 15.41 & 15.57 & 15.79 & 15.99 & 16.12 & 16.20 & 16.26 & 16.28 & 16.29 & 16.33 & 16.30 & 16.22 & 16.13 & 16.14 & 16.15 & 16.08 & 16.01 & 15.94 & 15.88 & 15.89 & 15.92 & 15.97 \\
\hline
\end{tabular}

Note: Only a portion of this table appears here. The complete table is available in ASCII format. 
Table T14. Spectral reflectance data, Hole 1101A.

\begin{tabular}{|c|c|c|c|c|c|c|c|c|c|c|c|c|c|c|c|c|c|c|c|c|c|}
\hline Core & $\begin{array}{l}\text { Core } \\
\text { type }\end{array}$ & Section & $\begin{array}{l}\text { Interval } \\
\text { top } \\
(\mathrm{cm})\end{array}$ & $\begin{array}{c}\text { Interval } \\
\text { bottom } \\
(\mathrm{cm})\end{array}$ & $\begin{array}{l}\text { Curator } \\
\text { depth } \\
\text { (mbsf) }\end{array}$ & $\begin{array}{l}\text { Recovery- } \\
\text { corrected } \\
\text { depth } \\
\text { (mbsf) }\end{array}$ & $\mathrm{L}^{*}$ & $a^{*}$ & $b^{*}$ & Munsell & Munsell & Munsell & 400 & 410 & 420 & 430 & 440 & 450 & 460 & 470 & 480 \\
\hline 1 & $\mathrm{H}$ & 1 & 2 & 2 & 0.02 & 0.02 & 4.65 & 48.84 & 10.66 & $0.8 \mathrm{Y}$ & $4.7 /$ & 1.5 & 10.91 & 11.11 & 11.37 & 11.73 & 12.11 & 12.51 & 12.88 & 13.21 & 13.54 \\
\hline 1 & $\mathrm{H}$ & 1 & 7 & 7 & 0.07 & 0.07 & 4.27 & 49.47 & 10.34 & $1.2 \mathrm{Y}$ & $4.7 /$ & 1.5 & 11.41 & 11.62 & 11.88 & 12.21 & 12.63 & 13.08 & 13.45 & 13.78 & 14.09 \\
\hline 1 & $\mathrm{H}$ & 1 & 12 & 12 & 0.12 & 0.12 & 4.54 & 48.48 & 10.61 & $1.0 \mathrm{Y}$ & $4.6 /$ & 1.5 & 10.76 & 10.95 & 11.20 & 11.54 & 11.91 & 12.30 & 12.66 & 12.98 & 13.31 \\
\hline 1 & $\mathrm{H}$ & 1 & 17 & 17 & 0.17 & 0.17 & 5.36 & 43.94 & 12.88 & $1.2 \mathrm{Y}$ & 4.21 & 1.8 & 7.32 & 7.50 & 7.73 & 8.06 & 8.43 & 8.84 & 9.20 & 09.51 & 9.84 \\
\hline 1 & $\mathrm{H}$ & 1 & 22 & 22 & 0.22 & 0.22 & 4.87 & 43.46 & 10.31 & $0.2 Y$ & $4.1 /$ & 1.4 & 8.25 & 8.39 & 8.60 & 8.90 & 9.20 & 9.49 & 9.76 & 10.01 & 10.24 \\
\hline 1 & $\mathrm{H}$ & 1 & 27 & 27 & 0.27 & 0.27 & 4.30 & 48.14 & 10.85 & $1.5 \mathrm{Y}$ & $4.6 /$ & 1.5 & 10.24 & 10.49 & 10.76 & 11.12 & 11.54 & 11.99 & 12.37 & 12.70 & 13.02 \\
\hline 1 & $\mathrm{H}$ & 1 & 32 & 32 & 0.32 & 0.32 & 4.01 & 48.66 & 12.50 & $2.7 Y$ & $4.6 /$ & 1.7 & 9.24 & 9.57 & 9.97 & 10.50 & 11.08 & 11.62 & 12.10 & 12.52 & 12.92 \\
\hline 1 & $\mathrm{H}$ & 1 & 37 & 37 & 0.37 & 0.37 & 3.09 & 51.19 & 9.96 & $2.9 \mathrm{Y}$ & $4.9 /$ & 1.4 & 12.06 & 12.42 & 12.80 & 13.32 & 13.89 & 14.43 & 14.84 & 15.20 & 15.57 \\
\hline 1 & $\mathrm{H}$ & 1 & 42 & 42 & 0.42 & 0.42 & 3.31 & 46.60 & 11.90 & $3.5 Y$ & $4.5 /$ & 1.6 & 8.03 & 8.43 & 8.87 & 9.43 & 10.04 & 10.63 & 11.11 & 11.50 & 11.86 \\
\hline
\end{tabular}

\begin{tabular}{cccccccccccccccccccccc}
\hline 490 & 500 & 510 & 520 & 530 & 540 & 550 & 560 & 570 & 580 & 590 & 600 & 610 & 620 & 630 & 640 & 650 & 660 & 670 & 680 & 690 & 700 \\
\hline 13.86 & 14.27 & 14.73 & 15.17 & 15.68 & 16.17 & 16.65 & 17.12 & 17.57 & 18.01 & 18.34 & 18.68 & 18.95 & 19.27 & 19.59 & 19.84 & 20.12 & 20.38 & 20.61 & 20.78 & 20.89 & 21.03 \\
14.44 & 14.83 & 15.29 & 15.75 & 16.27 & 16.76 & 17.22 & 17.71 & 18.12 & 18.54 & 18.89 & 19.13 & 19.41 & 19.68 & 19.94 & 20.16 & 20.39 & 20.62 & 20.83 & 20.99 & 21.08 & 21.21 \\
13.65 & 14.03 & 14.48 & 14.94 & 15.42 & 15.94 & 16.42 & 16.89 & 17.28 & 17.70 & 18.07 & 18.34 & 18.61 & 18.91 & 19.22 & 19.46 & 19.73 & 19.99 & 20.21 & 20.36 & 20.48 & 20.64 \\
10.22 & 10.62 & 11.06 & 11.51 & 12.03 & 12.53 & 12.99 & 13.48 & 13.92 & 14.32 & 14.66 & 14.99 & 15.28 & 15.58 & 15.88 & 16.16 & 16.44 & 16.67 & 16.88 & 17.07 & 17.22 & 17.33 \\
10.51 & 10.81 & 11.15 & 11.51 & 11.94 & 12.36 & 12.74 & 13.14 & 13.52 & 13.89 & 14.21 & 14.49 & 14.75 & 15.03 & 15.33 & 15.59 & 15.86 & 16.12 & 16.33 & 16.49 & 16.62 & 16.75 \\
13.38 & 13.79 & 14.27 & 14.73 & 15.23 & 15.72 & 16.18 & 16.66 & 17.07 & 17.43 & 17.77 & 18.06 & 18.30 & 18.56 & 18.80 & 19.02 & 19.23 & 19.44 & 19.64 & 19.78 & 19.90 & 20.02 \\
13.33 & 13.81 & 14.39 & 14.99 & 15.62 & 16.18 & 16.69 & 17.19 & 17.60 & 18.00 & 18.29 & 18.52 & 18.71 & 18.90 & 19.09 & 19.27 & 19.43 & 19.60 & 19.76 & 19.92 & 20.02 & 20.14 \\
15.96 & 16.40 & 16.91 & 17.41 & 17.98 & 18.49 & 18.94 & 19.36 & 19.70 & 20.05 & 20.28 & 20.47 & 20.61 & 20.76 & 20.91 & 21.04 & 21.15 & 21.30 & 21.44 & 21.55 & 21.64 & 21.75 \\
12.26 & 12.70 & 13.21 & 13.73 & 14.30 & 14.81 & 15.25 & 15.68 & 16.04 & 16.37 & 16.60 & 16.72 & 16.84 & 16.97 & 17.07 & 17.14 & 17.26 & 17.37 & 17.46 & 17.56 & 17.63 & 17.71 \\
\hline
\end{tabular}

Note: Only a portion of this table appears here. The complete table is available in ASCII format. 
T.C.W. WOLF-WELLING ET AL.

Data Report: Diffuse Spectral Reflectance Data

Table T15. Spectral reflectance data, Hole 1103A.

\begin{tabular}{|c|c|c|c|c|c|c|c|c|c|c|c|}
\hline Core & $\begin{array}{l}\text { Core } \\
\text { type }\end{array}$ & Section & $\begin{array}{l}\text { Interval } \\
\text { top }(\mathrm{cm})\end{array}$ & $\begin{array}{l}\text { Interval } \\
\text { bottom } \\
(\mathrm{cm})\end{array}$ & $\begin{array}{l}\text { Curator } \\
\text { Depth } \\
\text { (mbsf) }\end{array}$ & $L^{*}$ & $a^{*}$ & $b^{*}$ & Munsell & Munsell & Munsell \\
\hline 27 & $\mathrm{R}$ & 1 & 2 & 2.1 & 247.32 & 40.59 & 0.48 & 4.30 & $8.3 Y$ & $3.9 /$ & 0.7 \\
\hline 27 & $\mathrm{R}$ & 1 & 7 & 7.1 & 247.37 & 33.41 & 0.26 & 2.60 & $8.3 Y$ & 3.21 & 0.4 \\
\hline 27 & $\mathrm{R}$ & 1 & 12 & 12.1 & 247.42 & 41.59 & 0.08 & 2.58 & $9.3 Y$ & $4.0 /$ & 0.4 \\
\hline 27 & $\mathrm{R}$ & 1 & 17 & 17.1 & 247.47 & 46.08 & -0.19 & 2.38 & $1.0 \mathrm{GY}$ & $4.5 /$ & 0.4 \\
\hline 27 & $\mathrm{R}$ & 1 & 22 & 22.1 & 247.52 & 41.82 & 0.06 & 2.44 & $9.4 \mathrm{Y}$ & $4.1 /$ & 0.4 \\
\hline 27 & $\mathrm{R}$ & 1 & 27 & 27.1 & 247.57 & 41.82 & 0.17 & 2.68 & $8.7 Y$ & $4.0 /$ & 0.4 \\
\hline 27 & $\mathrm{R}$ & 1 & 32 & 32.1 & 247.62 & 39.16 & 0.44 & 3.18 & $7.4 \mathrm{Y}$ & $3.8 /$ & 0.5 \\
\hline 27 & $\mathrm{R}$ & 1 & 37 & 37.1 & 247.67 & 38.27 & 0.26 & 2.86 & $8.3 Y$ & $3.7 /$ & 0.4 \\
\hline 27 & $\mathrm{R}$ & 1 & 42 & 42.1 & 247.72 & 43.06 & -0.05 & 2.42 & $0.1 \mathrm{GY}$ & 4.21 & 0.4 \\
\hline 27 & $\mathrm{R}$ & 1 & 47 & 47.1 & 247.77 & 29.26 & 0.32 & 2.57 & $7.9 \mathrm{Y}$ & $2.8 /$ & 0.4 \\
\hline 27 & $\mathrm{R}$ & 1 & 52 & 52.1 & 247.82 & 26.54 & 0.54 & 2.72 & $6.5 Y$ & $2.6 /$ & 0.5 \\
\hline 27 & $\mathrm{R}$ & 1 & 57 & 57.1 & 247.87 & 38.09 & 0.34 & 3.08 & $8.0 \mathrm{Y}$ & $3.7 /$ & 0.5 \\
\hline 27 & $\mathrm{R}$ & 1 & 62 & 62.1 & 247.92 & 21.83 & 0.29 & 2.36 & $7.9 Y$ & $2.1 /$ & 0.5 \\
\hline 27 & $\mathrm{R}$ & 1 & 67 & 67.1 & 247.97 & 38.03 & 0.49 & 3.16 & $7.1 Y$ & $3.7 /$ & 0.5 \\
\hline 27 & $\mathrm{R}$ & 1 & 72 & 72.1 & 248.02 & 48.66 & 0.45 & 3.53 & $7.7 Y$ & $4.7 /$ & 0.5 \\
\hline 27 & $\mathrm{R}$ & 1 & 77 & 77.1 & 248.07 & 39.20 & 0.15 & 2.68 & $8.8 \mathrm{Y}$ & $3.8 /$ & 0.4 \\
\hline 27 & $\mathrm{R}$ & 1 & 82 & 82.1 & 248.12 & 42.94 & -0.05 & 2.14 & $0.1 \mathrm{GY}$ & 4.21 & 0.3 \\
\hline 27 & $\mathrm{R}$ & 1 & 87 & 87.1 & 248.17 & 40.09 & 0.16 & 2.75 & $8.8 \mathrm{Y}$ & $3.9 /$ & 0.4 \\
\hline 27 & $\mathrm{R}$ & 1 & 92 & 92.1 & 248.22 & 39.85 & 0.16 & 2.75 & $8.8 \mathrm{Y}$ & $3.9 /$ & 0.4 \\
\hline 27 & $\mathrm{R}$ & 1 & 97 & 97.1 & 248.27 & 34.91 & 0.12 & 2.53 & $9.0 \mathrm{Y}$ & $3.4 /$ & 0.4 \\
\hline 27 & $\mathrm{R}$ & 1 & 102 & 102.1 & 248.32 & 33.35 & 0.02 & 2.28 & $9.5 \mathrm{Y}$ & 3.21 & 0.4 \\
\hline 27 & $\mathrm{R}$ & 1 & 107 & 107.1 & 248.37 & 36.35 & 0.13 & 2.49 & $8.9 Y$ & $3.5 /$ & 0.4 \\
\hline 27 & $\mathrm{R}$ & 1 & 112 & 112.1 & 248.42 & 35.72 & 0.15 & 2.38 & $8.9 Y$ & $3.5 /$ & 0.4 \\
\hline 27 & $\mathrm{R}$ & 1 & 117 & 117.1 & 248.47 & 35.80 & 0.16 & 2.60 & $8.9 Y$ & $3.5 /$ & 0.4 \\
\hline 27 & $\mathrm{R}$ & 1 & 122 & 122.1 & 248.52 & 37.15 & -0.01 & 2.40 & $9.8 \mathrm{Y}$ & $3.6 /$ & 0.4 \\
\hline 27 & $\mathrm{R}$ & 1 & 127 & 127.1 & 248.57 & 39.53 & 0.07 & 2.58 & $9.4 \mathrm{Y}$ & $3.8 /$ & 0.4 \\
\hline 27 & $\mathrm{R}$ & 1 & 132 & 132.1 & 248.62 & 42.74 & -0.02 & 2.48 & $9.9 Y$ & $4.1 /$ & 0.4 \\
\hline 27 & $\mathrm{R}$ & 1 & 137 & 137.1 & 248.67 & 42.85 & 0.04 & 2.77 & $9.6 \mathrm{Y}$ & $4.1 /$ & 0.4 \\
\hline 27 & $\mathrm{R}$ & 1 & 142 & 142.1 & 248.72 & 43.90 & -0.03 & 2.53 & $0.1 \mathrm{GY}$ & $4.3 /$ & 0.4 \\
\hline 27 & $\mathrm{R}$ & 1 & 147 & 147.1 & 248.77 & 46.11 & -0.07 & 2.60 & $0.4 \mathrm{GY}$ & $4.5 /$ & 0.4 \\
\hline 27 & $\mathrm{R}$ & 2 & 2 & 2.1 & 248.82 & 25.96 & 0.14 & 1.95 & $8.4 \mathrm{Y}$ & $2.5 /$ & 0.4 \\
\hline 27 & $\mathrm{R}$ & 2 & 7 & 7.1 & 248.87 & 27.55 & 0.19 & 2.26 & $8.3 Y$ & $2.7 /$ & 0.4 \\
\hline 27 & $\mathrm{R}$ & 2 & 12 & 12.1 & 248.92 & 35.29 & -0.27 & 2.24 & $1.2 \mathrm{GY}$ & $3.4 /$ & 0.4 \\
\hline 27 & $\mathrm{R}$ & 2 & 17 & 17.1 & 248.97 & 36.29 & 0.31 & 2.80 & $7.9 Y$ & $3.5 /$ & 0.4 \\
\hline 27 & $\mathrm{R}$ & 2 & 22 & 22.1 & 249.02 & 39.46 & -0.38 & 1.52 & $3.0 \mathrm{GY}$ & $3.8 /$ & 0.3 \\
\hline 27 & $\mathrm{R}$ & 2 & 27 & 27.1 & 249.07 & 34.79 & 0.11 & 2.54 & $8.9 Y$ & $3.4 /$ & 0.4 \\
\hline 27 & $\mathrm{R}$ & 2 & 32 & 32.1 & 249.12 & 31.59 & 0.18 & 2.38 & $8.4 \mathrm{Y}$ & $3.1 /$ & 0.4 \\
\hline 27 & $\mathrm{R}$ & 2 & 37 & 37.1 & 249.17 & 28.44 & 0.23 & 2.42 & $8.2 Y$ & $2.8 /$ & 0.4 \\
\hline 27 & $\mathrm{R}$ & 2 & 42 & 42.1 & 249.22 & 28.92 & 0.18 & 2.27 & $8.4 \mathrm{Y}$ & $2.8 /$ & 0.4 \\
\hline 27 & $\mathrm{R}$ & 2 & 47 & 47.1 & 249.27 & 31.33 & -0.10 & 1.90 & $0.2 \mathrm{GY}$ & $3.0 /$ & 0.3 \\
\hline 27 & $\mathrm{R}$ & 2 & 52 & 52.1 & 249.32 & 23.83 & 0.15 & 1.91 & $8.2 \mathrm{Y}$ & $2.3 /$ & 0.4 \\
\hline 27 & $\mathrm{R}$ & 2 & 57 & 57.1 & 249.37 & 21.07 & 0.40 & 1.72 & $5.8 \mathrm{Y}$ & $2.0 /$ & 0.3 \\
\hline 27 & $\mathrm{R}$ & 2 & 62 & 62.1 & 249.42 & 21.75 & 0.35 & 1.79 & $6.3 Y$ & $2.1 /$ & 0.3 \\
\hline 27 & $\mathrm{R}$ & 2 & 67 & 67.1 & 249.47 & 22.15 & 0.27 & 1.95 & $7.2 Y$ & $2.1 /$ & 0.4 \\
\hline 27 & $\mathrm{R}$ & 2 & 72 & 72.1 & 249.52 & 21.38 & 0.31 & 1.66 & $6.6 \mathrm{Y}$ & $2.1 /$ & 0.3 \\
\hline 27 & $\mathrm{R}$ & 2 & 77 & 77.1 & 249.57 & 27.25 & 0.33 & 1.88 & $6.5 Y$ & $2.6 /$ & 0.3 \\
\hline 27 & $\mathrm{R}$ & 2 & 82 & 82.1 & 249.62 & 35.10 & 0.51 & 2.53 & $5.8 \mathrm{Y}$ & $3.4 /$ & 0.4 \\
\hline 27 & $\mathrm{R}$ & 2 & 87 & 87.1 & 249.67 & 46.85 & -0.35 & 1.33 & $3.3 \mathrm{GY}$ & $4.5 /$ & 0.3 \\
\hline 27 & $\mathrm{R}$ & 2 & 92 & 92.1 & 249.72 & 38.58 & -0.03 & 1.63 & $9.8 \mathrm{Y}$ & $3.7 /$ & 0.3 \\
\hline 27 & $\mathrm{R}$ & 2 & 97 & 97.1 & 249.77 & 39.73 & -0.01 & 2.02 & $9.6 \mathrm{Y}$ & $3.9 /$ & 0.3 \\
\hline 27 & $\mathrm{R}$ & 3 & 2 & 2.1 & 249.82 & 21.48 & 0.31 & 1.50 & $5.9 Y$ & $2.1 /$ & 0.3 \\
\hline 27 & $\mathrm{R}$ & 3 & 7 & 7.1 & 249.87 & 24.74 & 0.01 & 1.58 & $9.4 \mathrm{Y}$ & $2.4 /$ & 0.3 \\
\hline 27 & $\mathrm{R}$ & 3 & 12 & 12.1 & 249.92 & 26.04 & 0.24 & 1.52 & $6.8 \mathrm{Y}$ & $2.5 /$ & 0.3 \\
\hline 27 & $\mathrm{R}$ & 3 & 17 & 17.1 & 249.97 & 35.63 & -0.41 & 1.15 & $4.1 \mathrm{GY}$ & $3.5 /$ & 0.3 \\
\hline 27 & $\mathrm{R}$ & 3 & 22 & 22.1 & 250.02 & 31.16 & 0.36 & 2.00 & $6.5 Y$ & $3.0 /$ & 0.3 \\
\hline 27 & $\mathrm{R}$ & 3 & 27 & 27.1 & 250.07 & 31.57 & 0.30 & 2.03 & $7.0 Y$ & $3.1 /$ & 0.3 \\
\hline 27 & $\mathrm{R}$ & 3 & 32 & 32.1 & 250.12 & 37.72 & 0.50 & 2.72 & $6.3 Y$ & $3.7 /$ & 0.4 \\
\hline 27 & $\mathrm{R}$ & 3 & 37 & 37.1 & 250.17 & 31.51 & 0.65 & 2.71 & $5.2 Y$ & $3.1 /$ & 0.4 \\
\hline 27 & $\mathrm{R}$ & 3 & 42 & 42.1 & 250.22 & 28.11 & 0.33 & 2.48 & $7.5 Y$ & $2.7 /$ & 0.4 \\
\hline 27 & $\mathrm{R}$ & 3 & 47 & 47.1 & 250.27 & 32.67 & 0.37 & 2.26 & $6.7 Y$ & 3.21 & 0.4 \\
\hline 27 & $\mathrm{R}$ & 3 & 52 & 52.1 & 250.32 & 32.54 & 0.54 & 2.48 & $5.7 Y$ & 3.21 & 0.4 \\
\hline 27 & $\mathrm{R}$ & 3 & 57 & 57.1 & 250.37 & 35.87 & 0.39 & 2.74 & $6.9 Y$ & $3.5 /$ & 0.4 \\
\hline 27 & $\mathrm{R}$ & 3 & 62 & 62.1 & 250.42 & 30.21 & 0.51 & 2.40 & $5.7 Y$ & $2.9 /$ & 0.4 \\
\hline
\end{tabular}

Note: Only a portion of this table appears here. The complete table is available in ASCII format. 